\title{
Perturbative Analysis of Adaptive Smoothing Methods in Quantifying Large-Scale Structure
}

\author{
NAOKI SETO \\ Department of Physics, Faculty of Science, Kyoto University, Kyoto 606-8502, Japan
}

\begin{abstract}
Smoothing operation to make continuous density field from observed point-like distribution of galaxies is crucially important for topological or morphological analysis of the large-scale structure, such as, the genus statistics or the area statistics (equivalently the level crossing statistics). It has been pointed out that the adaptive smoothing filters are more efficient tools to resolve cosmic structures than the traditional spatially fixed filters. We study weakly nonlinear effects caused by two representative adaptive methods often used in smoothed hydrodynamical particle ( $\mathrm{SPH}$ ) simulations. Using framework of second-order perturbation theory, we calculate the generalized skewness parameters for the adaptive methods in the case of initially power-law fluctuations. Then we apply the multidimensional Edgeworth expansion method and investigate weakly nonlinear evolution of the genus statistics and the area statistics. Isodensity contour surfaces are often parameterized by the volume fraction of the regions above a given density threshold. We also discuss this parameterization method in perturbative manner.
\end{abstract}

Subject headings: cosmology: theory — large-scale structure of the universe

\section{Introduction}

The large-scale distribution of galaxies is one of the most important sources to study formation and evolution of cosmic structures. Now there are two ongoing large-scale redshift surveys of galaxies, the Sloan Digital Sky Survey (SDSS, Gunn \& Weinberg 1995) and the Anglo-Australian Telescope 2dF Survey (Colless 1998). These surveys will bring us enormous information of three-dimensional galaxy distribution and are expected to revolutionarily improve our knowledge of the large-scale structure in the universe.

The two-point correlation function of galaxies, or its Fourier transform, the power spectrum are simple as well as powerful tools, and have been widely used to quantify 
clustering of galaxies (e.g. Totsuji \& Kihara 1969, Peebles 1974, Davis \& Peebles 1983). These two quantities are based on the second-order moments of matter fluctuations. When the fluctuations are Random Gaussian distributed, the two-point correlation function or the power spectrum contains full statistical information of the fluctuations. Even though the initial seed of structure formation is often assumed to be random Gaussian distributed as predicted by the standard inflation scenarios (Guth \& Pi 1982, Hawking 1982, Starobinsky 1982), this simple assumption has not been observationally established. Moreover, the cosmic structures observed today are more or less affected by nonlinear gravitational evolution. Therefore, only with these two quantities, we cannot study the large-scale structure properly.

Other statistical methods have been proposed and is expected to play complimentary roles to the traditional analyses based on the second-order moment. The skewness parameter characterizes the asymmetry of one point probability distribution function (PDF) of the density field and has been investigated in deep (Peebles 1980, Fry 1984, Juszkiewicz, Bouchet \& Colombi 1993, Bernardeau 1994, Scoccimarro 1998, Seto 1999). Beside higher-order moments (such as skewness), there are other statistical approaches designed to directly measure the geometrical or morphological aspects of galaxy clustering. Connectivity of the isodensity contour is an interesting target for these approaches. For example, the genus statistics were proposed by Gott, Melott \& Dickinson (1986), the area statistics (equivalently the level crossing statistics) by Ryden (1988), and percolation analysis by Klypin (1988). In addition, the Minkowski functionals recently attain much attention (e.g. Minkowski 1903, Mecke, Buchert \& Wagner 1994, Schmalzing \& Buchert 1997, Kerscher et al. 1997).

To analyze observed cosmic structures, smoothing operation becomes crucially important in some cases. The observed galaxies are distributed in point-like manner, but geometrical or morphological analyses, such as, the genus statistics, are usually based on continuous (smoothed) density field (see also Babul \& Starkman 1992, Luo \& Vishniac 1995). We have traditionally used filters with spatially fixed smoothing radius for analyzing the large-scale structure. Even though this method is the simplest from theoretical point of views, other possibilities are worth investigated. The local statistical fluctuations due to the discreteness of particles are determined by the number of particles contained in the smoothing kernel. If we use a spatially fixed filter, we can measure smoothed quantities at overdensity regions relatively more accurately than at underdense regions. As a result, quality of information becomes inhomogeneous. This inhomogeneity is caused by the simple choice to spatially fix the smoothing radius. There must be more efficient methods to resolve cosmic structure from particle distribution (Hernquist \& Katz 1989). Actually, Springel et al. (1998) have pointed out that the signal to noise ratio of the genus statistics 
is considerably improved by using adaptive smoothing methods. Adaptive methods are based on Lagrangian description, use nearly same number of "particles" (mass elements) to construct smoothed density field (Hernquist \& Katz 1989) and are expected to be less affected by discreteness of mass elements. Therefore, it seems reasonable that we can resolve cosmic structures more efficiently, using these methods.

In this article, we perturbatively analyzed quantitative effects caused by adaptive smoothing methods. We pay special attention to three representative examples, the skewness parameter, the genus statistics and the area statistics. As the skewness is basically defined by the one point PDF, we can, in principle, discuss it without making continuous density field. But its analysis is very instructive to see nonlinear effects accompanied with adaptive smoothing methods. As a first step, we mainly study the density field in real space and do not discuss the effects of biasing (Kaiser 1984, Bardeen, Bond, Kaiser \& Szalay 1986, Dekel \& Lahav 1999).

This article is organized as follows. In $\S 2$ we describe basic properties of the adaptive smoothing and introduce its two main approaches. Then perturbative formulas are derived for each of them. In $\S 3$ we discuss the skewness parameters of the density field smoothed by these two adaptive methods. We evaluate them using second-order perturbation theory. Some of results in this section can be straightforwardly applied to the density field in the redshift space. Then we discuss weakly nonlinear effects of the genus and the area statistics using the multidimensional Edgeworth expansion method explored by Matsubara (1994). To characterize the isodensity contour, parameterization based on the volume fraction above a given density threshold is often adopted. In $\S 4.1$ we discuss this parameterization in perturbative manner. In $\S 4.2$ we explicitly evaluate the generalized skewness which is closely related to the genus and the area statistics. In $\S 4.3$ and $\S 4.4$, we show the weakly

nonlinear effects on these two statistics with various smoothing methods. We make a brief summary in $\S 5$.

\section{Adaptive Smoothing Method}

The (unsmoothed) density contrast field $\delta(\boldsymbol{x})$ at a point $\boldsymbol{x}$ is defined in terms of the mean density of the universe $\bar{\rho}$ and the local density $\rho(\boldsymbol{x})$ as

$$
\delta(\boldsymbol{x})=\frac{\rho(\boldsymbol{x})-\bar{\rho}}{\bar{\rho}} .
$$

In this article we assume that the primordial density fluctuations obey Random Gaussian distribution which is completely characterized by the (linear) matter power spectrum. 
Unless we state explicitly, we limit our analysis in the real space density field. But some of our results are straightforwardly applied to the redshift space quantities, as shown in the next section.

Isotropic filters with spatially constant smoothing radius $R$ have been traditionally used to obtain continuous smoothed density field $\delta_{F R}(\boldsymbol{x})$ as follows

$$
\delta_{F R}(\boldsymbol{x})=\int d \boldsymbol{x}^{\prime} \delta\left(\boldsymbol{x}^{\prime}\right) W\left(\left|\boldsymbol{x}^{\prime}-\boldsymbol{x}\right| ; R\right) .
$$

Here the function $W(\boldsymbol{x} ; R)$ is a spatial filter function and the subscript $F$ indicate the fixed smoothing. Most of theoretical analyses in the large-scale structure have been based on this fixed smoothing method. As for the functional shape of $W(\boldsymbol{x} ; R)$, two kinds of functions are often used (e.g. Bardeen et al. 1986, Matsubara 1995). One is the Gaussian filter and defined as

$$
W(\boldsymbol{x} ; R)=(2 \pi)^{-3 / 2} R^{-3} \exp \left(-\boldsymbol{x}^{2} / 2 R^{2}\right) .
$$

The other one is the top-hat filter and has a compact support as

$$
W(\boldsymbol{x} ; R)= \begin{cases}3 /\left(4 \pi R^{3}\right) & (|\boldsymbol{x}| \leq R) \\ 0 & (|\boldsymbol{x}|>R) .\end{cases}
$$

In this article we mainly use the Gaussian filter. This filter is useful for quantifying the large-scale structure from observed noisy data sets. In addition, algebraic manipulations for the Gaussian filter are generally much simpler than for the top-hat filter.

Next let us discuss the basic properties of adaptive smoothing methods (Hernquist \& Katz 1989, Thomas \& Couchman 1992, Springel et al. 1998). The essence of these methods is to change the smoothing radius $R$ as a function of position $\boldsymbol{x}$ according to its local density contrast. With a given spherically symmetric kernel $W$, we determine the smoothing radius $R(\boldsymbol{x})$ so that the total mass included within the kernel becomes constant.

$$
\bar{\rho} \int d \boldsymbol{x}^{\prime}\left(1+\delta\left(\boldsymbol{x}^{\prime}\right)\right) R(\boldsymbol{x})^{3} W\left(\left|\boldsymbol{x}^{\prime}-\boldsymbol{x}\right| ; R(\boldsymbol{x})\right)=\bar{\rho} R^{3} .
$$

The radius $R(\boldsymbol{x})$ becomes smaller than the standard value $R$ in a overdense region and becomes larger in a underdense region. In a system constituted by equal mass particles as in standard N-body simulations, the smoothing radius $R(\boldsymbol{x})$ is determined so that the total number of particles in a filter becomes constant. Thus adaptive smoothing is basically Lagrangian description and their smoothing radii are closely related to the resolution of spatial structures.

We can solve the variable smoothing radius $R(\boldsymbol{x})$ in equation (5) by perturbatively expanding the deviation $\delta R(\boldsymbol{x}) \equiv R(\boldsymbol{x})-R$. In this procedure we regard the density 
contrast $\delta$ as the order parameter of the perturbative expansion. After some calculations we obtain the first-order solution as follows

$$
\delta R(\boldsymbol{x})=-\frac{1}{3} \delta_{F R}(\boldsymbol{x}) R+O\left(\delta^{2}\right)
$$

This simple result seems quite reasonable with the relation below.

$$
R(\boldsymbol{x})^{3}\left(1+\delta_{F R}(\boldsymbol{x})\right)=R^{3}\left(1+O\left(\delta^{2}\right)\right) .
$$

This relation roughly shows that the total mass within the smoothing radius $R(\boldsymbol{x})$ does not depend on position $\boldsymbol{x}$.

With the variable smoothing radius $R(\boldsymbol{x})$ (solution for eq.[5]) we can practice adaptive smoothing. As pointed out by Hernquist \& Katz (1989) for the smoothed particle hydrodynamics (SPH), there exist two different methods (gather and scatter approaches) to assign the smoothed density contrast field at each point $\boldsymbol{x}$. The gather approach is simply use the solution $R(\boldsymbol{x})$ at the point $\boldsymbol{x}$ in interest and the smoothed field is formally written as

$$
\delta_{G R}(\boldsymbol{x})=\int d \boldsymbol{x}^{\prime} \delta\left(\boldsymbol{x}^{\prime}\right) W\left(\left|\boldsymbol{x}^{\prime}-\boldsymbol{x}\right| ; R(\boldsymbol{x})\right)-C(R),
$$

the subscript $G$ indicates the gather approach. In this case, the volume average of the first term in the right hand side dose not vanish and we have added a term $C(R)$ so that the total volume average of $\delta_{G R}(\boldsymbol{x})$ becomes zero.

In the scatter approach we use the solution $R\left(\boldsymbol{x}^{\prime}\right)$ for each point where a mass element exists. We can write down the smoothed field at $\boldsymbol{x}$ as

$$
\delta_{S R}(\boldsymbol{x})=\int d \boldsymbol{x}^{\prime} \delta\left(\boldsymbol{x}^{\prime}\right) W\left(\left|\boldsymbol{x}^{\prime}-\boldsymbol{x}\right| ; R\left(\boldsymbol{x}^{\prime}\right)\right),
$$

the subscript $S$ represents the scatter approach. In this case the volume average becomes zero. We only dilute the mass element at point $\boldsymbol{x}^{\prime}$ with the density profile proportional to $W\left(\left|\boldsymbol{x}^{\prime}-\boldsymbol{x}\right| ; R\left(\boldsymbol{x}^{\prime}\right)\right)$ around that point. Note that the spatial dependence of the smoothing radius $R(\cdot)$ is different between equations (8) and (9).

Next we evaluate equations for $\delta_{G R}(\boldsymbol{x})$ and $\delta_{S R}(\boldsymbol{x})$ up to second-order of the density contrast $\delta$ using perturbative solution of the smoothing radius $R(\boldsymbol{x})=R+\delta R(\boldsymbol{x})$ given in equation (6). The results are given as

$$
\begin{aligned}
\delta_{G R}(\boldsymbol{x}) & =\delta_{F R}(\boldsymbol{x})-\frac{1}{3} \delta_{F R}(\boldsymbol{x}) R \frac{\partial}{\partial R} \delta_{F R}(\boldsymbol{x})+\frac{1}{6} R \frac{d}{d R} \sigma_{R}^{2}+O\left(\delta^{3}\right) \\
\delta_{S R}(\boldsymbol{x}) & =\delta_{F R}(\boldsymbol{x})-\frac{R}{3} \int d \boldsymbol{x}^{\prime} \partial_{R} W\left(\left|\boldsymbol{x}^{\prime}-\boldsymbol{x}\right| ; R\right) \delta\left(\boldsymbol{x}^{\prime}\right) \delta_{F R}\left(\boldsymbol{x}^{\prime}\right)+O\left(\delta^{3}\right) .
\end{aligned}
$$


The formula for the scatter approach is somewhat complicated, compared with the gather approach. Also in numerical analysis, the scatter approach requires higher computational costs (Springel et al. 1998). This reflects nonlocal character of the smoothing radius.

Equations (10) and (11) show apparently that the corrections due to the adaptive methods start from second-order of $\delta$. Therefore, their effects are expected to be comparable to second-order (nonlinear) effects predicted by cosmological gravitational perturbation theory (Peebles 1980, Fry 1984, Goroff et al. 1986). Adaptive smoothing methods modify the quantities which characterize the nonlinear mode couplings, such as the skewness parameter of density field.

If we use the Gaussian filter, the leading-order correction for the scatter approach is expressed as follows

$$
\int \frac{d \boldsymbol{k}}{(2 \pi)^{3}} \frac{d \boldsymbol{l}}{(2 \pi)^{3}} \exp \left[-\frac{\left(2 \boldsymbol{l}^{2}+\boldsymbol{k}^{2}+2 \boldsymbol{k} \cdot \boldsymbol{l}\right) R^{2}}{2}\right] \delta(\boldsymbol{k}) \delta(\boldsymbol{l}) \exp [i(\boldsymbol{k}+\boldsymbol{l}) \cdot \boldsymbol{x}] \frac{(\boldsymbol{k}+\boldsymbol{l})^{2} R^{2}}{3},
$$

where $\delta(\boldsymbol{k})$ and $\delta(\boldsymbol{l})$ are the Fourier coefficients of the density contrast and defined as

$$
\delta(\boldsymbol{k})=\int d \boldsymbol{x} \delta(\boldsymbol{x}) \exp (-i \boldsymbol{k} \cdot \boldsymbol{x})
$$

Formula (12) is useful to quantitatively evaluate nonlinear effects caused by the scatter approach.

\section{Skewness}

In this section we investigate modifications of the skewness parameter $S$ caused by the two adaptive methods. Skewness is a fundamental quantities to characterize asymmetry of the one point PDF of the density field (Peebles 1980, Fry 1984, Juszkiewicz, Bouchet \& Colombi 1993, Bernardeau 1994). It is defined as

$$
S=\frac{\left\langle\delta^{3}\right\rangle}{\sigma^{4}}
$$

where the angular bracket $\langle\cdot\rangle$ represents to take the ensemble average and $\sigma\left(\equiv\left\langle\delta^{2}\right\rangle^{1 / 2}\right)$ is the rms fluctuation of $\delta$. Here, we discuss the leading-order contributions for the numerator $\left\langle\delta^{3}\right\rangle$ and denominator $\sigma^{4}$. As we have already commented in $\S 1$, the skewness parameter can be discussed without making continuous density field. It can be basically defined by the count probability distribution function, and spatial relation between one region and another one is unnecessary (e.g. Gaztañaga 1992, Bouchet et al. 1993, Kim \& Strauss 1998, and 
references therein, see also Colombi, Szapudi \& Szalay 1998). Therefore our effort in this article to resolve cosmic structures by using the adaptive smoothing might be irrelevant for observational determination of the skewness parameter. But perturbative analysis in this section is very useful to grasp nonlinear effects caused by the adaptive smoothing methods and become basis for studying statistics of isodensity contours such as the genus statistics or the area statistics discussed in the next section.

The leading-order contribution for the rms fluctuation $\sigma$ is written in terms of the linear (primordial) power spectrum $\left\langle\delta_{1}(\boldsymbol{k}) \delta_{1}(\boldsymbol{l})\right\rangle=(2 \pi)^{3} \delta_{D r c}(\boldsymbol{k}+\boldsymbol{l}) P(k)\left(\delta_{1}(\boldsymbol{k})\right.$ : linear mode, $\delta_{D r c}(\cdot)$ : Dirac's delta function). With the Fourier transformed filter function $w(k R)$ we have

$$
\sigma_{R}^{2}=\left\langle\delta_{R}^{2}(\boldsymbol{x})\right\rangle=\int \frac{d \boldsymbol{k}}{(2 \pi)^{3}} P(k) w(k R)^{2},
$$

where the suffix $R$ is added to explicitly indicate the smoothing radius $R$. Throughout in this article, we use power-law spectra $P(k)$ as

$$
P(k)=A k^{n}, \quad-3<k \leq 1
$$

for these scale-free models the normalization factor $A$ becomes irrelevant and we can simply put $A=1$ below. Furthermore, as shown later, the skewness parameter does not depend on the smoothing radius in our leading-order analysis. From equation (15) we have the variance $\sigma_{R}^{2}$ for the Gaussian filter as

$$
\sigma_{R}^{2}=\int_{0}^{\infty} \frac{d k}{2 \pi^{2}} k^{n+2} e^{-k^{2} R^{2}}=\frac{R^{-n-3}}{(2 \pi)^{2}} \Gamma\left(\frac{3+n}{2}\right) .
$$

The integral (15) logarithmically diverges for $n=-3$, but skewness $S$ is well-behaved in the limit $n \rightarrow-3$ from above. As it shows interesting behavior at this specific spectral index, we also discuss quantities at $n=-3$ regarding them as the limit values.

Calculation of the third-order moment $\left\langle\delta^{3}\right\rangle$ is more complicated than that of the variance $\sigma^{2}$ discussed so far. When the initial fluctuation is random Gaussian distributed as assumed in this article, the linear contribution for the third-order moment becomes exactly zero due to the symmetric distribution of the density contrast $\delta$ around the origin $\delta=0$. Nonlinear mode couplings induce asymmetry in this distribution. Therefore, we resort to higher-order perturbation theory. The leading-order contribution for the skewness parameter without smoothing operation is given by Peebles (1980) in the case of Einstein de-Sitter background as

$$
S=\frac{34}{7}
$$

It is convenient to use the Fourier space representation to calculate the third-order moment for the smoothed density field. Following the standard procedure, we expand a nonlinear 
Fourier modes of overdensity $\delta$ and the (irrotational) peculiar velocity field $\boldsymbol{V}$ as (Fry 1984, Goroff et al. 1986)

$$
\begin{aligned}
\delta(\boldsymbol{x}) & =\delta_{1}(\boldsymbol{x})+\delta_{2}(\boldsymbol{x})+\cdots, \\
\boldsymbol{V}(\boldsymbol{x}) & =\boldsymbol{V}_{1}(\boldsymbol{x})+\boldsymbol{V}_{2}(\boldsymbol{x})+\cdots,
\end{aligned}
$$

where $\delta_{1}(\boldsymbol{x})$ and $\boldsymbol{V}_{1}(\boldsymbol{x})$ are the linear modes and $\delta_{2}(\boldsymbol{x})$ and $\boldsymbol{V}_{2}(\boldsymbol{x})$ the second-order modes. We perturbatively solve the continuity, Euler and Poisson equations,

$$
\begin{aligned}
\frac{\partial}{\partial t} \delta(\boldsymbol{x})+\frac{1}{a} \nabla[\boldsymbol{V}(\boldsymbol{x})\{1+\delta(\boldsymbol{x})\}] & =0, \\
\frac{\partial}{\partial t} \boldsymbol{V}(\boldsymbol{x})+\frac{1}{a}[\boldsymbol{V}(\boldsymbol{x}) \cdot \nabla] \boldsymbol{V}(\boldsymbol{x})+\frac{\partial_{t} a}{a} \boldsymbol{V}(\boldsymbol{x})+\frac{1}{a} \nabla \phi(\boldsymbol{x}) & =0, \\
\nabla^{2} \phi(\boldsymbol{x})-4 \pi a^{2} \rho(t) \delta(\boldsymbol{x}) & =0,
\end{aligned}
$$

where $a$ represents the scale factor. The second-order solution in $\boldsymbol{k}$-space is given as

$$
\delta_{2}(\boldsymbol{k})=\int \frac{d \boldsymbol{l}}{(2 \pi)^{3}} \delta_{1}(\boldsymbol{l}) \delta_{1}(\boldsymbol{k}-\boldsymbol{l}) J(\boldsymbol{l}, \boldsymbol{k}-\boldsymbol{l}),
$$

or in $\boldsymbol{x}$-space

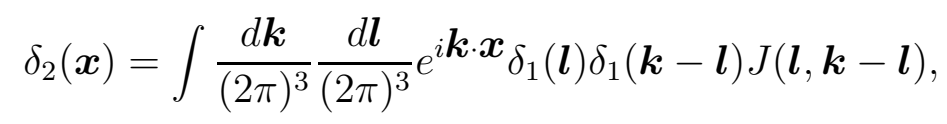

where the kernel $J$ is defined by

$$
J(\boldsymbol{k}, \boldsymbol{l})=\frac{1}{2}(1+K)+\frac{\boldsymbol{k} \cdot \boldsymbol{l}}{2}\left(\frac{1}{k^{2}}+\frac{1}{l^{2}}\right)+\frac{1}{2}(1-K) \frac{(\boldsymbol{k} \cdot \boldsymbol{l})^{2}}{k^{2} l^{2}} .
$$

The factor $K(\Omega, \lambda)$ weakly depends on the density parameter $\Omega$ and cosmological constant $\lambda$ as shown in the fitting formula (Matsubara 1995, see also Bouchet et al. 1992)

$$
K(\Omega, \lambda) \simeq \frac{3}{7} \Omega^{-1 / 30}-\frac{\lambda}{80}\left(1-\frac{3}{2} \lambda \log _{10} \Omega\right) .
$$

In the ranges of two parameters $\Omega$ and $\lambda$

$$
0.1 \leq \Omega \leq 1, \quad 0.1 \leq \lambda \leq 1,
$$

the difference of $K(\Omega, \lambda)$ from $K=3 / 7$ is within $8 \%$. Therefore, in the following analysis we basically study the Einstein de-Sitter background and use $K=3 / 7$.

Using the second-order solution (21) we can derive the well known formula for the third-order moment as follows (Juszkiewicz et al. 1993)

$$
\left\langle\delta_{R}^{3}\right\rangle=6 \int \frac{d \boldsymbol{k}}{(2 \pi)^{3}} \frac{d \boldsymbol{l}}{(2 \pi)^{3}} P(k) P(l) J(\boldsymbol{k}, \boldsymbol{l}) w(k R) w(l R) w(|\boldsymbol{k}+\boldsymbol{l}| R) .
$$


Let us simplify this six-dimensional integral $d \boldsymbol{k} d \boldsymbol{l}$. In the case of the Gaussian filter

$$
w(k R)=\exp \left(-k^{2} R^{2} / 2\right),
$$

we can change $\left\langle\delta_{R}^{3}\right\rangle$ to the following form (Matsubara 1994)

$$
\left\langle\delta_{R}^{3}\right\rangle=\frac{3}{28 \pi^{4}}\left(5 I_{220}+7 I_{131}+2 I_{222}\right)
$$

where we have defined

$$
I_{a b c}=\int_{0}^{\infty} d k \int_{0}^{\infty} d l \int_{-1}^{1} d u \exp \left[-R^{2}\left(k^{2}+l^{2}+u k l\right)\right] k^{a} l^{b} u^{c} P(k) P(l) .
$$

For a power-law initial fluctuation $P(k)=k^{n}$, we obtain a final closed formula (Matsubara 1994, Lokas et al. 1995)

$$
S_{F}(n)=3 F\left(\frac{n+3}{2}, \frac{n+3}{2}, \frac{3}{2} ; \frac{1}{4}\right)-\left(n+\frac{8}{7}\right) F\left(\frac{n+3}{2}, \frac{n+3}{2}, \frac{5}{2} ; \frac{1}{4}\right),
$$

where $F$ is the Hypergeometric function.

In the case of the top-hat filter whose Fourier transform is given by

$$
w(k R)=\frac{3}{(k R)^{3}}(\sin k R-k R \cos k R),
$$

the final form of $S$ becomes very simple as follows (Juszkiewicz et al. 1993, Bernardeau 1994)

$$
S_{F}(n)=\frac{34}{7}-(n+3) .
$$

This formula is not only valid for pure power-law initial fluctuations but also for general power spectra with effective spectral index defined at the smoothing radius $R$ as

$$
n \equiv-\frac{d \ln \sigma_{R}^{2}}{d \ln R}-3 .
$$

Equations (29) and (31) are only the leading-order contribution and more higher-order effects might change them considerably. Thus it is quite important to compare these analytic formulas with fully nonlinear numerical simulations and clarify validity of the perturbative formulas. There are many works on this topic and the analytic predictions show surprisingly good agreement with numerical simulations, even at $\sigma \sim 1$ (e.g. Baugh, Gaztañaga \& Efstathiou 1995, Hivon et al. 1995, Juszkiewicz et al. 1995, Łokas et al. 1995). 
So far we have discussed skewness $S$ with fixed smoothing methods. For the third-order moments $\left\langle\delta_{R}^{3}\right\rangle$, the second-order effects caused by the gravitational evolution and that caused by the adaptive smoothing are decoupled, as we can see from equations (10) and (11). Thus we can write the skewness parameter for adaptive methods in the following forms

$$
\begin{aligned}
& S_{G}=S_{F}+\Delta S_{G}, \\
& S_{S}=S_{F}+\Delta S_{S} .
\end{aligned}
$$

Here $\Delta S_{G}$ and $\Delta S_{S}$ are the correction terms caused by the adaptive smoothing methods. In the next two subsections we calculated these terms explicitly.

\subsection{Gather Approach}

First we calculate the correction term $\Delta S_{G}$ for the gather approach. With equation (10) this term is easily transformed to the following equation (see Appendix A.1 for derivation)

$$
\Delta S_{G}=-\frac{d \ln \sigma_{R}^{2}}{d \ln R} .
$$

For a power-law spectrum we have simple equation below

$$
\Delta S_{G}(n)=(n+3) .
$$

In derivation of equation (35) we only use Gaussianity of the one point PDF of the linear smoothed field $\delta_{R}$. Therefore, these formulas do not depend on the shape of the smoothing filter nor the cosmological parameters $\Omega$ or $\lambda$. Furthermore they are valid also in the redshift space, if we use the the distant observer approximation. Thus equation (35) has strong predictability.

Hivon et al. (1995) perturbatively examined the skewness parameters in redshift space and evaluate them both for the top-hat filter and the Gaussian filter. They also compared their analytic results with numerical results. They found that these two show agreement only in the range $\sigma \lesssim 0.1$, in contrast to the skewness parameter in the real space $\sigma \lesssim 1.0$. They commented that this limitation is mainly due to the finger of god effects (e.g. Davis \& Peebles 1983).

Here we use their analytic results and combine our new formula with them. In figure 1 we present the skewness parameters for various spectral indexes $n$ both in the real and redshift spaces. For simplicities we limit our analysis for the Einstein de-Sitter background. 
For the Gaussian filter, the skewness parameter by the gather method is a increasing function of spectral index $n$ both in real and redshift spaces. This dependence is contrast to the skewness with the fixed smoothing method. Comparing the skewness in the real and the redshift spaces, $n$ dependence of the gather method is somewhat weaker in real space than in redshift space, but this tendency is also different from the fixed smoothing.

For the top-hat filter, there is no spectral index $n$ - dependence in the real space. We have $S=34 / 7$ which is the same as the unsmoothed value (Peebles 1980). Bernardeau (1994) pointed out that the skewness $S$ filtered with the top-hat filter in Lagrangian space does not depend on the power spectrum and is given by $S=34 / 7$. As the adaptive smoothing is basically Lagrangian description, this fact seems reasonable. In the case of the redshift space we have a fitting formula below

$$
S_{G}(n)=\frac{35.2}{7}-0.15(n+3), \quad(\text { Einstein de Sitter background })
$$

which is based on formula (49) of Hivon et al (1995). Again $n$ dependence is very weak and becomes weaker for $\Omega<1$ (see Fig.4 of Hivon et al. 1995). Finally, we comment the possibility that our perturbative treatment of the redshift space skewness becomes worse in the adaptive methods than in the fixed smoothing method. In the adaptive methods, smoothing radius of a high density region becomes smaller and the (strongly nonlinear) finger of god effects might not be suppressed well.

\subsection{Scatter Approach}

Next we calculate the correction term $\Delta S_{S}$ for the scatter approach. We only discuss the real space density field smoothed with the Gaussian filter (eq.[3]). From equation (12) we obtain the following equation (see Appendix A.1),

$$
\Delta S_{S R}(n) \sigma_{R}^{4}=2 \int \frac{d \boldsymbol{k}}{(2 \pi)^{3}} \frac{d \boldsymbol{l}}{(2 \pi)^{3}} \exp \left[-\frac{\left(3 \boldsymbol{l}^{2}+2 \boldsymbol{k}^{2}+2 \boldsymbol{k} \cdot \boldsymbol{l}\right) R^{2}}{2}\right] P(k) P(l)(\boldsymbol{k}+\boldsymbol{l})^{2} R^{2} .
$$

The six dimensional integral $d \boldsymbol{k} d \boldsymbol{l}$ is simplified to a three dimensional integral $d k d l d u$ as in equations (25) and (28). Then we have the following relation

$$
\begin{aligned}
\Delta S_{S R}(n) \sigma_{R}^{4}= & \frac{1}{4 \pi^{4}} \int_{0}^{\infty} d k \int_{0}^{\infty} d l \int_{-1}^{1} d u \exp \left[-\frac{\left(3 l^{2}+2 k^{2}+2 k l u\right) R^{2}}{2}\right] \\
& \times k^{2} l^{2} P(k) P(l)\left(k^{2}+l^{2}+2 k l u\right) R^{2}
\end{aligned}
$$

For a pure-power law fluctuation we obtain the following analytic formula

$$
\Delta S_{S}(n)=-2^{(n+3) / 2} 3^{-(n+5) / 2}(n+3)\left\{\frac{2}{3}(n+3) F\left(\frac{5+n}{2}, \frac{5+n}{2}, \frac{5}{2}, \frac{1}{6}\right)\right.
$$




$$
\left.-5 F\left(\frac{3+n}{2}, \frac{5+n}{2}, \frac{3}{2}, \frac{1}{6}\right)\right\} .
$$

In contrast to the previous gather approach, this result is valid only to the real space skewness with the Gaussian filter. In table 1 we present numerical values of $\Delta S_{S}(n)$. In figure 2 we show $S_{S}(n)$ as a function of the spectral index $n$. We can see that $n$ dependence is similar to the gather approach but now it becomes weaker. If we change $n$ from -3 to -1 , skewness $S$ changes $\sim 25 \%$ for the scatter approach, $\sim 45 \%$ for the gather approach, and $\sim 38 \%$ for the fixed smoothing.

\section{Statistics of Isodensity Contour}

The genus number is a topological quantity and defined by the number of the homotopy classes of closed curves that may be drawn on a surface without cutting them into two pieces. This definition seems highly mathematical, but there are more intuitive methods to count the genus number. First one is to notice the number of holes and isolated regions of the surface in interest. Second one is to count stationary points of the surface along one spatial direction (Adler 1981, Bardeen et al. 1986). With these equivalent methods, we can calculate the genus density as follows

$$
\begin{aligned}
\text { Genus density } & =\frac{N(\text { holes })-N(\text { isolated regions })}{\text { volume }} \\
& =-\frac{N(\text { maxima })+N(\text { minima })-N(\text { saddle points })}{2 \times \text { volume }} .
\end{aligned}
$$

For example, in the case of one-sphere, we have $N$ (holes $)=0$ and $N$ (isolated regions) $=1$ and genus number becomes -1 . We obtain the same result with equation (42). The genus number density of isodensity contour of the large-scale structure is a powerful measure to quantify connectivity of galaxy clustering, such as, filamentary networks, sheet-like or bubble-like structures. The genus density of a high density contours is expected to be negative as the surfaces would show disconnected meatball-like structure. But the genus density for contours around the mean density $\delta \sim 0$ would be positive as they would look like highly connected sponge-like structure (Gott, Melott \& Dickinson 1986). The genus density as a function of the matter density threshold is called the genus statistics and has been widely investigated both numerically and observationally (Gott, Weinberg \& Melott 1987, Weinberg, Gott \& Melott 1987, Melott, Weinberg \& Gott 1988, Gott et al. 1989, Park \& Gott 1991, Park, Gott \& da Costa 1992, Weinberg \& Cole 1992, Moore et al. 1992, Vogeley, Park, Geller, Huchra \& Gott 1994, Rhoads, Gott \& Postman 1994, Matsubara \& Suto 1996, Coles, Davies \& Pearson 1996, Sahni et al. 1997, Protogeros \& Weinberg 1997, 
Coles, Pearson, Borgani, Plionis \& Moscardini 1998, Canavezes et al. 1998, Springel et al. 1998)

Usually we use the local expression (42) to analytically study the genus statistics. For the genus density of isodensity contour at $\nu \equiv \delta / \sigma$, this expression is written as follows (Doroshkevich 1970, Adler 1981, Bardeen et al. 1986, Hamilton, Gott \& Weinberg 1986)

$$
G(\nu)=-\frac{1}{2}\left\langle\delta_{D r c}[\delta(\boldsymbol{x})-\nu \sigma] \delta_{D r c}\left[\partial_{1} \delta(\boldsymbol{x})\right] \delta_{D r c}\left[\partial_{2} \delta(\boldsymbol{x})\right]\left|\partial_{3} \delta(\boldsymbol{x})\right|\left(\partial_{11} \delta(\boldsymbol{x}) \partial_{22} \delta(\boldsymbol{x})-\partial_{12} \delta(\boldsymbol{x})^{2}\right)\right\rangle,
$$

where $\delta_{D r c}(\cdot)$ represents the Dirac's delta function. The first one $\delta_{D r c}[\delta(\boldsymbol{x})-\nu \sigma]$ specifies the contour $\nu \equiv \delta / \sigma$. The second and third ones $\delta_{D r c}\left[\partial_{1} \delta(\boldsymbol{x})\right], \delta_{D r c}\left[\partial_{2} \delta(\boldsymbol{x})\right]$ specify the stationary points along $x_{3}$ direction. The term $\left(\partial_{11} \delta(\boldsymbol{x}) \partial_{22} \delta(\boldsymbol{x})-\partial_{12} \delta(\boldsymbol{x})^{2}\right)$ is the determinant of the Hesse-matrix and assigns proper signatures for the stationary points corresponding to signs of equation (42). Even though equation (43) introduces a specific spatial direction ( $x_{3}$-axis), Seto et al. (1997) derived a rotationarilly symmetric formula, and studied nonlinear evolution of the genus statistics using the Zeldovich approximation (Zeldovich 1970)

In the case of an isotropic random Gaussian fluctuation which is usually assumed as the initial condition of the structure formation, the complicated formula (43) is simplified to (Doroshkevich 1970, Adler 1981, Bardeen et al. 1986, Hamilton, Gott \& Weinberg 1986)

$$
G(\nu)=\frac{1}{(2 \pi)^{2}}\left(\frac{\sigma_{1}^{2}}{3 \sigma^{2}}\right)^{3 / 2} e^{-\nu^{2} / 2}\left(1-\nu^{2}\right)
$$

where $\sigma_{1}^{2}$ is defined as

$$
\sigma_{1}^{2}=\left\langle(\nabla \delta)^{2}\right\rangle=\frac{1}{2 \pi^{2}} \int_{0}^{\infty} d k P(k) k^{4} w(k R)^{2} .
$$

Nonlinear evolution of the genus statistics had been studied using N-body simulations, but most analytical predictions for the genus statistics have been based on the linear formula (44). To compare it with observed distribution of galaxies we have to use sufficiently large smoothing radius to reduce nonlinearities. However, such a large smoothing radius is not statistically preferable for the finiteness of our survey volume.

Matsubara (1994) improved this difficulty by taking into account of weakly nonlinear effects in the genus statistics (see also Hamilton 1988, Okun 1990, Matsubara \& Yokoyama 1996, Seto et al. 1997). He used the multidimensional Edgeworth expansion method and added the first-order nonlinear correction to the linear formula. His result is written as

$$
G(\nu)=-\frac{1}{(2 \pi)^{2}}\left(\frac{\sigma_{1}^{2}}{3 \sigma^{2}}\right)^{3 / 2} e^{-\nu^{2} / 2}\left[H_{2}(\nu)+\sigma\left(\frac{S}{6} H_{5}(\nu)+\frac{3 T}{2} H_{3}(\nu)+3 U H_{1}(\nu)\right)+O\left(\sigma^{2}\right)\right] .
$$


This formula is valid for statistically isotropic and homogeneous weakly random Gaussian fields. Here functions $H_{n}(\nu) \equiv(-1)^{n} e^{\nu^{2} / 2}(d / d \nu)^{n} e^{-\nu^{2} / 2}$ are the Hermite polynomials,

$$
\begin{aligned}
& H_{0}(x)=1, \quad H_{1}(x)=x, \quad H_{2}(x)=x^{2}-1, \\
& H_{3}(x)=x^{3}-3 x, \quad H_{5}(x)=x^{5}-10 x^{3}+15 x .
\end{aligned}
$$

In equation (46), $S$ is the skewness parameter discussed in the previous section. $T$ and $U$ are called the generalized skewness parameters and defined by

$$
\begin{aligned}
T & =-\frac{1}{2 \sigma^{2} \sigma_{1}^{2}}\left\langle\delta^{2} \Delta \delta\right\rangle \\
U & =-\frac{3}{4 \sigma_{1}^{4}}\langle\nabla \delta \cdot \nabla \delta \Delta \delta\rangle .
\end{aligned}
$$

Matsubara \& Suto (1996) examined the perturbative formula (46) using N-body simulations. For power-law spectra with $n=1,0$ and -1 , they found that this formula are in reasonable agreement with numerical results in the range $-0.2 \lesssim \nu \sigma \lesssim 0.4$.

Next let us briefly summarize the area statistics $N_{3}(\nu)$ which were proposed by Ryden (1988) and investigated detailedly by Ryden et al. (1989). The area statistics are defined as the mean area of isodensity contour surface per unit volume. For statistically homogeneous and isotropic fluctuations, the area statistics are equal to twice the mean number of isodensity contour crossings along a straight line of unit length. These two statistics are thus equivalent (beside factor 2), but the contour crossing statistics are easier to compute numerically (Ryden 1988).

As in equation (43), the area statistics for isodensity contour $\delta=\nu \sigma$ is written as

$$
N_{3}(\nu)=\left\langle\delta_{D r c}[\delta(\boldsymbol{x})-\nu \sigma]|\nabla \delta(\boldsymbol{x})|\right\rangle
$$

In the case of isotropic Random Gaussian fluctuations, we have the following formula (Ryden 1988)

$$
N_{3}(\nu)=\frac{2}{\pi}\left(\frac{\sigma_{1}^{2}}{3 \sigma^{2}}\right)^{1 / 2} e^{-\nu^{2} / 2}
$$

Weakly nonlinear effects on the area statistics can be discussed with a similar technique used to derive equation (46) (Matsubara 1995). In this case, we need information of the density field up to its first spatial derivative and nonlinear correction is expressed in terms of two parameters $S$ and $T$ as follows

$$
N_{3}(\nu)=\frac{2}{\pi}\left(\frac{\sigma_{1}^{2}}{3 \sigma^{2}}\right)^{1 / 2} e^{-\nu^{2} / 2}\left[1+\sigma\left(\frac{S}{6} H_{3}(\nu)+\frac{T}{2} H_{1}(\nu)\right)+O\left(\sigma^{2}\right)\right] .
$$


In the rest of this section we consider nonlinear effects of the adaptive smoothing methods on the genus and the area statistics. We calculate the generalized skewness parameters $T$ and $U$ both for the gather approach and the scatter approach. We limit our analysis to the real space density field smoothed by Gaussian filters.

\subsection{Reparameterization of Isodensity Contour}

Equation (46) is weakly non-Gaussian genus density for isodensity contour surfaces parameterized by the simple definition $\nu=\delta / \sigma$. There is another conventional method to name contour surfaces. In this method, we notice the volume fraction $f$ above the density threshold of the contour in interest (e.g. Gott, Melott \& Dickinson 1986, Gott et al. 1989), and parameterize the contour using value $\nu_{r}$ defined by

$$
\nu_{r} \equiv \operatorname{erf}^{-1}(f)
$$

where the suffix $r$ indicates "reparameterization" and $\operatorname{erf}(x)$ is the error function defined by

$$
\operatorname{erf}(x) \equiv \frac{1}{\sqrt{2 \pi}} \int_{x}^{\infty} d y e^{-y^{2} / 2}
$$

This procedure is a kind of Gaussianization. Two methods coincide $\nu=\nu_{r}$ when the one point $\operatorname{PDF} P(\nu)$ is Gaussian distributed. If we use this new parameterization, the genus curve is apparently invariant under a monotonic mapping of the density contrast field $\delta$. Furthermore, it has been long known that the genus curve with $\nu_{r}$ parameterization (51) nearly keeps its original symmetric shape (eq.[44]) in the course of weakly nonlinear gravitational evolution of density field (e.g. Springel et al. 1998 and references therein). Almost the same kind of tendency has been confirmed for the area statistics (Ryden et al. 1989). Weakly nonlinear area density with $\nu_{r}$ parameterization remains at its linear shape very well. Here, let us relate these two parameterization methods for weakly nonlinear regime. Using the Edgeworth expansion method, the one point $\operatorname{PDF} P(\nu)$ is written in terms of the skewness $S$ up to the first-order nonlinear correction

$$
P(\nu)=\frac{1}{\sqrt{2 \pi}} e^{-\nu^{2} / 2}\left[1+\frac{\sigma S}{6} H_{3}(\nu)+O\left(\sigma^{2}\right)\right] .
$$

Juszkiewicz et al. (1995) examined this approximation using N-body simulations. They found that the above formula is accurate until $\sigma S$ reaches 1 . Therefore the inequality

$$
\sigma \lesssim S^{-1} \sim 0.2
$$


would be a standard for the validity of the perturbative analysis in this subsection. The volume fraction $f(\nu)$ above the threshold $\delta=\nu \sigma$ is given by

$$
f(\nu)=\int_{\nu}^{\infty} d x P(x)=\operatorname{erf}(\nu)+\frac{\sigma S}{6 \sqrt{2 \pi}}\left\{e^{-\nu^{2}}\left(\nu^{2}-1\right)\right\}+O\left(\sigma^{2}\right) .
$$

With equations (51) and (55) we obtain correspondence between $\nu$ and $\nu_{r}$ as follows

$$
\nu=\nu_{r}+\frac{\sigma S}{6}\left\{\nu_{r}^{2}-1\right\}+O\left(\sigma^{2}\right)
$$

Finally the genus density $G_{r}\left(\nu_{r}\right)$ in this new parameterization is given by $G(\nu)=G_{r}\left(\nu_{r}\right)$ and written as

$$
\begin{aligned}
G_{r}\left(\nu_{r}\right)= & -\frac{1}{(2 \pi)^{2}}\left(\frac{\sigma_{1}^{2}}{3 \sigma^{2}}\right)^{3 / 2} e^{-\nu_{r}^{2} / 2}\left[H_{2}\left(\nu_{r}\right)+\sigma\left(H_{3}(\nu)\left(-S+\frac{3}{2} T\right)\right.\right. \\
& \left.\left.+H_{1}(\nu)(-S+3 U)\right)+O\left(\sigma^{2}\right)\right] .
\end{aligned}
$$

Similarly we have the following result for the area statistics

$$
N_{3 r}\left(\nu_{r}\right)=\frac{2}{\pi}\left(\frac{\sigma_{1}^{2}}{3 \sigma^{2}}\right)^{1 / 2} e^{-\nu_{r}^{2} / 2}\left[1+\sigma\left(-\frac{S}{3}+\frac{T}{2}\right) H_{1}\left(\nu_{r}\right)+O\left(\sigma^{2}\right)\right] .
$$

In this case, the first nonlinear correction is simply proportional to $\nu_{r}$ (see eq.[47]) and it completely vanishes when we have $S=3 / 2 T$.

Later in $\S 4.3$ and $\S 4.4$, we will confirm that the nonlinear correction (proportional to $\sigma$ ) for the genus and area statistics with the fixed smoothing method are very small for $\nu_{r}$ parameterization, as experientally known in N-body simulations. In the followings, we use these two parameterizations $\nu$ and $\nu_{r}$.

\subsection{Generalized Skewness}

The generalized skewness $T$ and $U$ are basic ingredients to perturbatively evaluate the weakly non-Gaussian effects on the genus and the area statistics. For the Gaussian filter with a fixed smoothing radius, explicit formulas valid for the power-law initial fluctuations were derived by Matsubara (1994). They are given as follows

$$
\begin{aligned}
T_{F R}= & 3 F\left(\frac{n+3}{2}, \frac{n+5}{2}, \frac{3}{2}, \frac{1}{4}\right)-\left(n+\frac{18}{7}\right) F\left(\frac{n+3}{2}, \frac{n+5}{2}, \frac{5}{2}, \frac{1}{4}\right) \\
& +\frac{4(n-2)}{105} F\left(\frac{n+3}{2}, \frac{n+5}{2}, \frac{7}{2}, \frac{1}{4}\right), \\
U_{F R}= & F\left(\frac{n+5}{2}, \frac{n+5}{2}, \frac{5}{2}, \frac{1}{4}\right)-\frac{7 n+16}{35} F\left(\frac{n+5}{2}, \frac{n+5}{2}, \frac{7}{2}, \frac{1}{4}\right) .
\end{aligned}
$$


As shown in the case of the skewness parameter $S$ analyzed in $\S 3.1$ and $\S 3.2$, the second-order (first nonlinear) effects caused by the adaptive smoothing methods are decoupled from those induced by gravitational mode couplings. Thus we can calculate them separately and express the total values in forms similar to equations (33) and (34).

First, we analyze correction terms $\Delta T_{G R}$ and $\Delta U_{G R}$ for the gather approach. We define these terms by the following equations

$$
T_{G R}=T_{F R}+\Delta T_{G R}, \quad U_{G R}=U_{F R}+\Delta U_{G R} .
$$

After some tedious algebra using equation (10), we obtain the leading-order correction terms as follows (Appendix A.2)

$$
\begin{aligned}
\Delta T_{G R} & =\frac{2}{3}(n+4), \\
\Delta U_{G R} & =\frac{1}{3}(n+5) .
\end{aligned}
$$

These results are similar to the correction term for the skewness $\Delta S_{G R}=n+3$ (eq.[36]) which does not depend on the shape of the filter function. However, situation is not so much simple here. For the Gaussian filter we have the following relation,

$$
R \frac{\partial \delta_{R}(\boldsymbol{x})}{\partial R}=R^{2} \Delta_{x} \delta_{R}(\boldsymbol{x})
$$

This relation plays important roles to derive equations (61) and (62). But it does not hold for general filter functions and the simple results given in equations (61) and (62) are specific to the Gaussian filter. We summarize numerical data for the parameters $T_{G R}$ and $U_{G R}$ in Table 3.

Generalized skewness for the gather approach is much more complicated. If we write down them in the form

$$
T_{S R}=T_{S R}+\Delta T_{S R}, \quad U_{S R}=U_{S R}+\Delta U_{S R}
$$

correction terms $\Delta T_{S R}$ and $\Delta U_{S R}$ are written in the manner similar to equation (39) as follows (Appendix A.3)

$$
\begin{aligned}
-2 \Delta T_{S R}(n) \sigma_{R}^{2} \sigma_{1 R}^{2}= & \frac{1}{6 \pi^{4}} \int_{0}^{\infty} d k \int_{0}^{\infty} d l \int_{-1}^{1} d u \exp \left[-\frac{\left(3 l^{2}+2 k^{2}+2 k l u\right) R^{2}}{2}\right] \\
& \times k^{2} l^{2} P(k) P(l)\left(k^{2}+l^{2}+2 k l u\right)\left(k^{2}+l^{2}+k l u\right) R^{2}, \\
-\frac{4}{3} \Delta U_{S R}(n) \sigma_{1 R}^{4}= & \frac{1}{6 \pi^{4}} \int_{0}^{\infty} d k \int_{0}^{\infty} d l \int_{-1}^{1} d u \exp \left[-\frac{\left(3 l^{2}+2 k^{2}+2 k l u\right) R^{2}}{2}\right] \\
& \times k^{4} l^{4} P(k) P(l)\left(k^{2}+l^{2}+k l u\right)\left(1-u^{2}\right) R^{2} .
\end{aligned}
$$


We can calculate them explicitly as follows

$$
\begin{aligned}
\Delta T_{S R}=2^{(n+5) / 2} 3^{-(n+9) / 2}\left[4(n+3) F\left(\frac{n+5}{2}, \frac{n+5}{2}, \frac{5}{2}, \frac{1}{6}\right)\right. & \\
& +\frac{5}{2}(n+3)(n+5) F\left(\frac{n+5}{2}, \frac{n+7}{2}, \frac{5}{2}, \frac{1}{6}\right) \\
& -\frac{13(n+5)}{2} F\left(\frac{n+3}{2}, \frac{n+7}{2}, \frac{3}{2}, \frac{1}{6}\right) \\
& \left.-12(n+3) F\left(\frac{n+5}{2}, \frac{n+5}{2}, \frac{3}{2}, \frac{1}{6}\right)\right], \\
\Delta U_{S R}=-2^{(n+5) / 2} 3^{-(n+5) / 2}\left[4 F\left(\frac{n+5}{2}, \frac{n+5}{2}, \frac{5}{2}, \frac{1}{6}\right)\right. & +\frac{5(n+5)}{9} F\left(\frac{n+5}{2}, \frac{n+7}{2}, \frac{5}{2}, \frac{1}{6}\right) \\
& \left.-4 F\left(\frac{n+5}{2}, \frac{n+5}{2}, \frac{3}{2}, \frac{1}{6}\right)\right] .
\end{aligned}
$$

We present numerical data of the parameters $T_{S R}$ and $U_{S R}$ in Table 4 . Note that the magnitude of generalized skewness $T$ and $U$ becomes very small in the scatter approach. This fact becomes important in the next subsection.

\subsection{Weakly Nonlinear Genus Statistics}

In figures 3 to 5 , we show the weakly nonlinear genus density smoothed by three different methods (fixed, gather and scatter), using two types of parameterizations $\nu$ and $\nu_{r}$. All of these curves are smoothed by the Gaussian filter (eq.[3]). We plot the normalized genus curves $G(\nu) / G(0)$ or $G_{r}\left(\nu_{r}\right) / G_{r}(0)$ to see deviation from the symmetric linear genus curve $\propto\left(1-\nu^{2}\right) \exp \left(-\nu^{2} / 2\right)$. Upper panel of figure 3 is essentially same as Fig.1 of Matsubara (1994).

First, comparing upper and bottom panels of Fig.3, we can confirm the fact experientally known in N-body simulations. Weakly nonlinear genus curves for the fixed smoothing method are very close to the linear symmetric shape in the case of $\nu_{r}$ parameterization (e.g. Springel et al. 1998). For a spectral index with $n \sim-1$, three curves for $\sigma=0,0.2$ and 0.4 are nearly degenerated.

In Fig. 4 we present genus curves with the gather smoothing. From Figs.3 and 4 it is apparent that deviations from the linear curves become larger in the gather approach,

\footnotetext{
${ }^{1}$ Note that the amplitude of $G(0)$ or $G_{r}(0)$ are not changed by the first-order correction of $\sigma$.
} 
especially in tail parts. But these deviations become smaller with using parameter $\nu_{r}$.

Bottom panel of Fig.4 is calculated under conditions (gather approach and parameter $\nu_{r}$ ) similar to Fig.7 of Springel et al. (1998) which is obtained from N-body simulations. However overall shapes of these two are different. The minimum value of $G_{r}\left(\nu_{r}\right)$ are attained around the point $\nu_{r} \sim 1.5$ in our result, but this point is $\nu_{r} \sim-1.5$ in theirs. This difference might be caused by the difference of adopted filter function. We use the Gaussian filter but a different kernel (a spline kernel that is often used in SPH simulations) is adopted in their calculation (Monaghan \& Lattanzio 1985).

In figure 5 we show results for the scatter approach. Nonlinear effects are more prominent than two cases analyzed earlier. As shown in equations (46) and (56), nonlinear correction of the genus curves are written by combination of terms proportional to parameters $S, T$ and $U$. Some of their contribution cancel out, as realized in the case of the fixed or gather smoothing methods. However, amplitude of parameters $T$ and $U$ for the scatter approach becomes very small (see Table 4), and cancellation becomes weaker.

Based on the definition of the genus density (eq.[41]), nonlinear evolution of isodensity contours is sometimes described with such terminologies as, sponge-like (connected topology) or meatball like (disconnected topology). In the case of Random Gaussian initial fluctuations, linear theory predicts symmetry of the genus statistics with respect to the sign of density contrast $\delta$, and geometry of both high and low density tails look meat-ball like with negative genus density. If we use the fixed or gather smoothing methods, nonlinear effects make the genus number of a high density contour (e.g. $\nu=2)$ smaller, and topology of that region becomes more meatball-like (see figures 3 and 4). In contrast, contour of low density threshold (e.g. $\nu=-2$ ) is transformed in the direction of sponge-like topology, as quantified by the increase of genus number density (see figures 3 and 4).

It is not easy to understand behaviors of Fig. 5 for the scatter approach. But, changing point of views, we can discuss characters of this approach by comparing figures for various smoothing methods. To characterize nonlinear effects accompanied with the scatter approach, we apply the topological interpretation mentioned in the last paragraph. As shown in Fig.6 where various smoothing methods are compared, the scatter approach makes low density regions more meatball-like. But high density regions are transformed in the opposite direction. This trend shows remarkable contrast to the nonlinear gravitational effects traced by the simple fixed smoothing method. 


\subsection{Weakly Nonlinear Area Statistics}

In Figs.7 to 9, we plot the weakly nonlinear area statistics, using equation (50) for $\nu$ parameterization and equation (57) for $\nu_{r}$ parameterization. As in the analysis of the genus statistics, we normalize amplitude of the area density by $N_{3}(0)$ or $N_{3 r}(0)$. Comparing upper and bottom panels of figure 7 , it is apparent that $\nu_{r}$ parameterization is very effective to keep the original linear shape against weakly nonlinear effects. This fact has been confirmed experimentally in N-body simulations (Ryden et al. 1989) and is quite similar to the situation in the genus statistics explained in previous subsection. With $\nu_{r}$ parameterization (bottom panel of Fig.7), three curves for $\sigma=0,0.2$ and 0.4 are almost completely overlapped for all spectral indexes $n$. This fact seems reasonable as we have $S_{F} \simeq 3 / 2 T_{F}$ for the fixed smoothing method (see eq.[57] and Tables 1,3).

If we use the adaptive smoothing methods, weakly nonlinear correction on $N_{3}(\nu)(\nu$ parameterization) are considerable as shown in upper panels of Figs.8 and 9. This correction becomes apparently smaller for the gather approach, but not for the scatter approach. We have already commented that the first-order nonlinear correction for the area statistics is characterized by two parameters $S$ and $T$. For the scatter approach, $T$ parameter is very small for spectral indexes $n>-1$, and cancellation mentioned in $\S 4.3$ is not effective.

\section{Summary}

Observational analysis of galaxy clustering is one of the central issues in modern cosmology. Various methods have been proposed to quantify the clustering, and many of them (e.g. topological analyses of isodensity contour) are based on continuous smoothed density field. However what we can observe directly is distribution of point-like galaxies. Thus smoothing operation is crucially important in the analyses of the large-scale structure. From theoretical point of views, filters with spatially constant smoothing radii are natural

choice and have been widely adopted so far. But we should notice that there are no strong convincing reasons to stick to this traditional method.

There are few galaxies in void regions even at semi-nonlinear scales. In these regions density field obtained with fixed smoothing radius might be considerably affected by the discreteness of (point-like) mass elements, and might hamper our analyses of the cosmic structures. Adaptive smoothing is basically Lagrangian description, and we use nearly same number of particles to construct smoothed density field at each point. Thus it is quite possible that the adaptive methods are more efficient than the fixed methods to analyze the large-scale structure. Actually, Springel et al. (1998) have recently pointed out that 
using adaptive filters, signal to noise ratio of the genus statistics is improved even at weakly nonlinear scales $\gtrsim 10 h^{-1} \mathrm{Mpc}$.

In this article, we have developed a perturbative analysis of adaptive smoothing methods that are applied to quantify the large-scale structure. Even though adaptive methods might be promising approaches in observational cosmology, this kind of analytic investigation has not been done so far. Our targets are weakly nonlinear effects induced by two typical adaptive approaches, the gather approach and the scatter approach (Hernquist \& Katz 1989). The concept of these methods is easily understood with equations (8) and (9). The gather approach is easier to handle analytically. Numerical costs dealing with discrete particles' systems are also lower in this approach (Springel et al. 1998). Effects caused by these two adaptive methods start from second-order of $\delta$ in perturbative sense. They modify quantities which characterize the nonlinear mode couplings induced by gravity (e.g. Peebles 1980).

In $\S 3$ we have investigated the skewness parameter $S$ which is a fundamental measure to quantify asymmetry of one point PDF. We have shown that the skewness for a gather top-hat filter does not depend on the spectral index $n$ in real space, and very weakly depends on it ( $S \simeq 35.2-0.15 n$ : Einstein de-Sitter background) in redshift space. In the case of Gaussian filter, the skewness parameters show similar behaviors both in the scatter and gather approaches. They are increasing functions of $n$, in contrast to the fixed smoothing method.

Next in $\S 4$, the genus and area statistics have been studied with Gaussian adaptive filters. Our analysis is based on the multidimensional Edgeworth expansion explored by Matsubara (1994). We use two quantities $\nu(\equiv \delta / \sigma)$ and $\nu_{r}$ to parameterize isodensity contours. The latter $\nu_{r}$ is defined by the volume fraction above a given density threshold (Gott, Melott, \& Dickinson 1986). It is explicitly shown that using this parameterization, two statistics with the fixed smoothing method are very weakly affected by semi-nonlinear gravitational dynamics, as experientally confirmed by N-body simulations. For the gather smoothing, we found that the $\nu_{r^{-}}$parameterization is more effective to keep original linear shape of the area statistics than of the genus statistics.

The parameters $S, T$ and $U$ which characterize the nonlinear corrections of isodensity contour depend largely on the filtering methods. We can characterize nonlinear effects of these methods in somewhat intuitive manner, using results for the genus statistics. The scatter approach makes low density tails more meatball-like, but high density tails are transformed in the direction of sponge-like (connected) topology. This is a remarkable difference from fixed or gather smoothing methods. 
Our investigation in this article has been fully analytical one, using perturbative technique of cosmological density field. Numerical analyses based on N-body simulations would play complementary roles to results obtained here, and thus are very important. Apart from numerical investigations, perturbative treatment given in this article would be also developed further in several ways. The smoothed velocity field is crucially important material in observational cosmology as it is supposed to be less contaminated by effects of biasing (Dekel 1994, Strauss \& Willick 1995). But our observational information is limited to the line of sight peculiar velocities only at points where astrophysical objects exist. Thus construction of smoothed velocity field contains similar characters as discussed in this article (e.g. Bernardeau \& van de Weygaert 1996). There is another (more technical) problem that has not mentioned so far. In this article we have only studied spherically symmetric filter functions. Springel et al. (1998) have shown that signal to noise ratio of the genus curves is further improved by using a triaxial kernel, taking account of tensor information of local density field. This point must be also worth studying.

The author would like to thank J. Yokoyama for discussion and an anonymous referee for useful comments. He also thanks H. Sato and N. Sugiyama for their continuous encouragement. This work was partially supported by the Japanese Grant in Aid for Science Research Fund of the Ministry of Education, Science, Sports and Culture No. 3161. 


\section{A. Derivations of Parameters}

In this appendix we derive expressions for the correction terms $\Delta S, \Delta T$ and $\Delta U$ given in the main text. First we perturbatively expand the density contrast field smoothed by an adaptive filter as

$$
\delta_{A}(\boldsymbol{x})=\delta_{1}(\boldsymbol{x})+\delta_{2}(\boldsymbol{x})+\delta_{2 A}(\boldsymbol{x})+\cdots,
$$

where $\delta_{1}(\boldsymbol{x})$ is the linear mode, $\delta_{2}(\boldsymbol{x})$ is the second-order mode induced by gravity, and $\delta_{2 A}$ is the second-order correction term caused by an adaptive smoothing (the suffix $A$ represents "adaptive"). Then the third-order moment for $\delta_{A}(\boldsymbol{x})$ is given as

$$
\left\langle\delta_{A}^{3}\right\rangle=3\left\langle\delta_{2} \delta_{1}^{2}\right\rangle+3\left\langle\delta_{2 A} \delta_{1}^{2}\right\rangle+\cdots
$$

Thus the first-order correction term for the third-order moment is given as

$$
3\left\langle\delta_{2 A} \delta_{1}^{2}\right\rangle \text {. }
$$

In the same manner we have the following correction terms for $\left\langle\delta_{A}^{2} \nabla^{2} \delta_{A}\right\rangle$ and $\left\langle\nabla \delta_{A} \cdot \nabla \delta_{a} \nabla^{2} \delta_{A}\right\rangle$ as

$$
\begin{aligned}
& \left\langle\delta_{1}^{2} \nabla^{2} \delta_{2 A}\right\rangle+2\left\langle\delta_{1} \delta_{2 A} \nabla^{2} \delta_{1}\right\rangle \\
& \left\langle\nabla \delta_{1} \cdot \nabla \delta_{1} \nabla^{2} \delta_{2 A}\right\rangle+2\left\langle\nabla \delta_{1} \nabla \delta_{2 A} \nabla^{2} \delta_{1}\right\rangle .
\end{aligned}
$$

We can write down the second-order correction terms $\delta_{2 A}$ for the gather and scatter approaches with smoothing radius $R$ (see eqs. [10] and [11])

$$
\begin{aligned}
\delta_{2 G R}(\boldsymbol{x}) & =-\frac{1}{3} \delta_{1 R}(\boldsymbol{x}) R \frac{\partial}{\partial R} \delta_{1 R}(\boldsymbol{x})+\frac{1}{6} R \frac{d}{d R} \sigma_{R}^{2}, \\
\delta_{2 S R}(\boldsymbol{x}) & =-\frac{R}{3} \int d \boldsymbol{x}^{\prime} \partial_{R} W\left(\left|\boldsymbol{x}^{\prime}-\boldsymbol{x}\right| ; R\right) \delta_{1}\left(\boldsymbol{x}^{\prime}\right) \delta_{1 R}\left(\boldsymbol{x}^{\prime}\right) .
\end{aligned}
$$

where $\delta_{1 R}(\boldsymbol{x})$ represents the smoothed linear mode, $W\left(\left|\boldsymbol{x}^{\prime}-\boldsymbol{x}\right| ; R\right)$ is a filter function. The variance $\sigma_{R}$ of the matter fluctuations is given as

$$
\begin{aligned}
\sigma_{R}^{2} & =\left\langle\delta_{1 R}^{2}\right\rangle+O\left(\delta^{4}\right) \\
& =\int \frac{d \boldsymbol{k}}{(2 \pi)^{3}} w(k R)^{2} P(k)+O\left(\delta^{4}\right),
\end{aligned}
$$

where $w(k R)$ is a Fourier transformed filter function.

For the Gaussian filter (see eq.[3]) the above equations are given with the linear Fourier modes $\delta_{1}(\boldsymbol{k})$ as

$$
\delta_{2 G R}(\boldsymbol{x})=\int \frac{d \boldsymbol{k}}{(2 \pi)^{3}} \frac{d \boldsymbol{l}}{(2 \pi)^{3}} \exp \left[-\frac{\left(\boldsymbol{l}^{2}+\boldsymbol{k}^{2}\right) R^{2}}{2}\right] \delta_{1}(\boldsymbol{k}) \delta_{1}(\boldsymbol{l}) \frac{\boldsymbol{k}^{2} R^{2}}{3} \exp [i(\boldsymbol{k}+\boldsymbol{l}) \cdot \boldsymbol{x}]
$$




$$
\begin{aligned}
& +\frac{1}{6} R \frac{d}{d R} \sigma_{R}^{2}, \\
\delta_{2 S R}(\boldsymbol{x})= & -\int \frac{d \boldsymbol{k}}{(2 \pi)^{3}} \frac{d \boldsymbol{l}}{(2 \pi)^{3}} \exp \left[-\frac{\left(2 \boldsymbol{l}^{2}+\boldsymbol{k}^{2}+2 \boldsymbol{k} \cdot \boldsymbol{l}\right) R^{2}}{2}\right] \delta_{1}(\boldsymbol{k}) \delta_{1}(\boldsymbol{l}) \frac{(\boldsymbol{k}+\boldsymbol{l})^{2} R^{2}}{3} \\
& \times \exp [i(\boldsymbol{k}+\boldsymbol{l}) \cdot \boldsymbol{x}],
\end{aligned}
$$

Next we comment on the ensemble average of variables. We assume that the linear Fourier modes of density fluctuation are random Gaussian distributed. If variables $\{A, B, C, D\}$ obeys multivariable Gaussian distribution, we generally have the following relation

$$
\langle A B C D\rangle=\langle A B\rangle\langle C D\rangle+\langle A C\rangle\langle B D\rangle+\langle A D\rangle\langle B C\rangle .
$$

For the linear Fourier modes the above equation becomes

$$
\begin{aligned}
\left\langle\delta_{1}(\boldsymbol{k}) \delta_{1}(\boldsymbol{l}) \delta_{1}(\boldsymbol{m}) \delta_{1}(\boldsymbol{n})\right\rangle= & (2 \pi)^{6} P(k) P(l) \delta_{D r c}(\boldsymbol{k}+\boldsymbol{m}) \delta_{D r c}(\boldsymbol{l}+\boldsymbol{n}) \\
& +(2 \pi)^{6} P(k) P(m) \delta_{D r c}(\boldsymbol{k}+\boldsymbol{l}) \delta_{D r c}(\boldsymbol{m}+\boldsymbol{n}) \\
& +(2 \pi)^{6} P(k) P(l) \delta_{D r c}(\boldsymbol{k}+\boldsymbol{n}) \delta_{D r c}(\boldsymbol{l}+\boldsymbol{m}) .
\end{aligned}
$$

Here $\delta_{D r c}(\cdot)$ is the Dirac's delta function and $P(k)$ is the matter power spectrum. We evaluate expressions (A3)-(A5) using relations (A12)-(A13).

\section{A.1. Skewness}

For the gather approach the real-space representation (A6) is more convenient. Using property (A12) we obtain the following result

$$
\begin{aligned}
\left\langle\delta_{2 S R} \delta_{1 R}^{2}\right\rangle & =\left\langle-\delta_{1 R}^{3}\left(R \frac{\partial}{\partial R} \delta_{1 R}\right)\right\rangle+\frac{1}{2}\left\langle\delta_{1 R}^{2}\right\rangle \frac{d}{d R} \sigma_{R}^{2} \\
& =-3 \sigma_{1 R}^{2}\left\langle\delta_{1 R} \frac{\partial}{\partial R} \delta_{1 R}\right\rangle+\frac{1}{2} \sigma_{R}^{2} \frac{d}{d R} \sigma_{R}^{2} \\
& =-\sigma_{R}^{2} \frac{d}{d R} \sigma_{R}^{2} .
\end{aligned}
$$

The correction term for the skewness $S_{G}$ is written as equation (35)

$$
\Delta S_{G}=\frac{\left\langle\delta_{2 G R} \delta_{1 R}^{2}\right\rangle}{\sigma_{R}^{4}}=-\frac{1}{\sigma_{R}^{2}} \frac{d}{d R} \sigma_{R}^{2}=-\frac{d \ln \sigma_{R}^{2}}{d \ln R} .
$$

For power-law models we have a simple relation $\sigma_{R}^{2} \propto R^{-n-3}$, and the above expression becomes

$$
\Delta S_{G}=(n+3) .
$$


Here we should notice that relations (A17) and (A18) do not depend on the choice of filter functions.

Next let us evaluate the correction term for the skewness parameter in the case of the scatter approach. In this case we limit our analysis for a Gaussian filter (eq.[3]). From equation (A11) we have

$$
\begin{aligned}
3\left\langle\delta_{2 S R}(\boldsymbol{x}) \delta_{1 R}(\boldsymbol{x})^{2}\right\rangle= & \int \frac{d \boldsymbol{k}}{(2 \pi)^{3}} \frac{d \boldsymbol{l}}{(2 \pi)^{3}} \frac{d \boldsymbol{m}}{(2 \pi)^{3}} \frac{d \boldsymbol{n}}{(2 \pi)^{3}}(\boldsymbol{k}+\boldsymbol{l})^{2} R^{2} \\
& \exp \left[-\frac{\left(2 \boldsymbol{l}^{2}+\boldsymbol{k}^{2}+\boldsymbol{m}^{2}+\boldsymbol{n}^{2}+2 \boldsymbol{k} \cdot \boldsymbol{l}\right) R^{2}}{2}\right] \\
& \times\left\langle\delta_{1}(\boldsymbol{k}) \delta_{1}(\boldsymbol{l}) \delta_{1}(\boldsymbol{m}) \delta_{1}(\boldsymbol{n})\right\rangle \exp [i(\boldsymbol{k}+\boldsymbol{l}+\boldsymbol{m}+\boldsymbol{n}) \cdot \boldsymbol{x}] .
\end{aligned}
$$

Using equation (A13) we can simplify the above integral as

$$
2 \int \frac{d \boldsymbol{k}}{(2 \pi)^{3}} \frac{d \boldsymbol{l}}{(2 \pi)^{3}} \exp \left[-\frac{\left(3 \boldsymbol{l}^{2}+2 \boldsymbol{k}^{2}+2 \boldsymbol{k} \cdot \boldsymbol{l}\right) R^{2}}{2}\right] P(k) P(l)(\boldsymbol{k}+\boldsymbol{l})^{2} R^{2} .
$$

Note that the integrad of this expression depends only on the information of the shape of the triangle determined by two vectors $\boldsymbol{k}$ and $\boldsymbol{l}$. This triangle is characterized by three quantities, namely, two sides $k=|\boldsymbol{k}|, l=|\boldsymbol{l}|$ and cosine between them $u \equiv \boldsymbol{k} \cdot \boldsymbol{l} /(k l)$ with $-1 \leq u \leq 1$. We change variables from $\{\boldsymbol{k}, \boldsymbol{l}\}$ to $\{k, l, u\}$. The volume element is deformed as

$$
d \boldsymbol{k} d \boldsymbol{l} \Rightarrow 8 \pi^{2} d k d l d u .
$$

Thus we obtain equation (39). This equation looks somewhat complicated. For power-law models, however, we can easily evaluate it using mathematica (Wolfram 1996) and finally obtain analytical expression (40) given in the main text.

\section{A.2. Generalized Skewness for the Gather Approach}

For this approach we have the following relation for a Gaussian filter

$$
R \frac{\partial}{\partial R} \delta_{1 R}(\boldsymbol{x})=R^{2} \nabla^{2} \delta_{1 R}(\boldsymbol{x}) .
$$

Therefore the correction terms (A4) and (A5) can be written by combinations of the following five variables 4

$$
\left\{\delta, \nabla \delta, \nabla^{2} \delta, \nabla^{3} \delta, \nabla^{4} \delta\right\}
$$

\footnotetext{
${ }^{2}$ In this subsection we denote $\delta_{1 R}(\boldsymbol{x})$ simply by $\delta$.
} 
For example, equation (A4) is written as

$$
-\frac{1}{3} R^{2}\left[\left\langle\delta^{2} \nabla^{2}\left(\delta \nabla^{2} \delta\right)\right\rangle+2\left\langle\delta^{2}\left(\nabla^{2} \delta\right)\left(\nabla^{2} \delta\right)\right\rangle-2\left\langle\delta \nabla^{2} \delta\right\rangle\left\langle\delta \nabla^{2} \delta\right\rangle\right]
$$

Using property (A12), the above expression is deformed as

$$
-\frac{1}{3} R^{2}\left[3\left\langle\nabla^{2} \delta \nabla^{2} \delta\right\rangle\langle\delta \delta\rangle+2\left\langle\nabla \delta \nabla^{3} \delta\right\rangle\langle\delta \delta\rangle+3\left\langle\delta \nabla^{4} \delta\right\rangle\langle\delta \delta\rangle+4\left\langle\delta \nabla^{2} \delta\right\rangle^{2}\right]
$$

The moments appeared in the above equation can be written in terms of $P(k)$ as

$$
\begin{aligned}
-\left\langle\delta \nabla^{2} \delta\right\rangle & =\langle\nabla \delta \nabla \delta\rangle=\int \frac{d k}{2 \pi^{2}} k^{4} P(k) e^{-k^{2} R^{2}} \\
\left\langle\nabla^{2} \delta \nabla^{2} \delta\right\rangle & =-\left\langle\nabla^{3} \delta \nabla \delta\right\rangle=\left\langle\nabla^{4} \delta \delta\right\rangle=\int \frac{d k}{2 \pi^{2}} k^{6} P(k) e^{-k^{2} R^{2}}
\end{aligned}
$$

For a power-law models $\left(P(k) \propto k^{n}\right)$. These integrals are evaluated respectively as

$$
\sigma_{R}^{2} R^{-2}\left(\frac{n+3}{2}\right), \quad \sigma_{R}^{2} R^{-4}\left(\frac{n+3}{2}\right)\left(\frac{n+5}{2}\right) .
$$

With the definition of $T$ parameter (eq.[48]) we obtain the final result that is given in equation (61) as

$$
\Delta T_{G R}=\frac{2}{3}(n+4)
$$

To calculate the correction term (A5), let us use the Fourier space representation (A10). It is straightforward to get

$$
\begin{aligned}
\left\langle\nabla \delta_{1} \cdot \nabla \delta_{1} \nabla^{2} \delta_{2 A}\right\rangle+2\left\langle\nabla \delta_{1} \nabla \delta_{2 A} \nabla^{2} \delta_{1}\right\rangle= & \int \frac{d \boldsymbol{k}}{(2 \pi)^{3}} \frac{d \boldsymbol{l}}{(2 \pi)^{3}} \frac{d \boldsymbol{m}}{(2 \pi)^{3}} \frac{d \boldsymbol{n}}{(2 \pi)^{3}} \frac{k^{2} R^{2}}{3} \\
& \times \exp \left[-\frac{\left(\boldsymbol{l}^{2}+\boldsymbol{k}^{2}+\boldsymbol{m}^{2}+\boldsymbol{n}^{2}\right) R^{2}}{2}\right] \\
& \times\left\langle\delta_{1}(\boldsymbol{k}) \delta_{1}(\boldsymbol{l}) \delta_{1}(\boldsymbol{m}) \delta_{1}(\boldsymbol{n})\right\rangle \\
& \times \exp [i(\boldsymbol{k}+\boldsymbol{l}+\boldsymbol{m}+\boldsymbol{n}) \cdot \boldsymbol{x}], \\
& \times\left[-(\boldsymbol{m} \cdot \boldsymbol{n})(\boldsymbol{k}+\boldsymbol{l})^{2}\right. \\
& -\left(\boldsymbol{m} \cdot(\boldsymbol{k}+\boldsymbol{l}) \boldsymbol{n}^{2}\right)-\left(\boldsymbol{n} \cdot(\boldsymbol{k}+\boldsymbol{l}) \boldsymbol{m}^{2}\right)(\mathrm{A} 30
\end{aligned}
$$

\footnotetext{
${ }^{3}$ We obtain the same result starting from equation (A6).
} 
With equation (A13), the above integral becomes

$$
\begin{aligned}
& -\frac{1}{3} \int \frac{d \boldsymbol{k}}{(2 \pi)^{3}} \frac{d \boldsymbol{l}}{(2 \pi)^{3}} k^{2} R^{2} \exp \left[-\left(\boldsymbol{l}^{2}+\boldsymbol{l}^{2}\right) R^{2}\right] P(k) P(l)\left[4 k^{2} l^{2}-4(\boldsymbol{k} \cdot \boldsymbol{l})^{2}\right] \\
= & -\frac{1}{6 \pi^{4}} \int_{0}^{\infty} d k \int_{0}^{\infty} d l \int_{-1}^{1} d u \exp \left[-\left(l^{2}+k^{2}\right) R^{2}\right] k^{6} l^{4} P(k) P(l)\left(1-u^{2}\right) R^{2} \\
= & -\frac{2}{9 \pi^{4}} \int_{0}^{\infty} k^{6} P(k) \exp \left[-k^{2} R^{2}\right] \int d l l^{4} P(l) \exp \left[-l^{2} R^{2}\right] .
\end{aligned}
$$

For power-law models this expression becomes (see eqs.[A26]-[A28])

$$
-\frac{8}{9} \sigma_{R}^{4}\left(\frac{n+3}{2}\right)^{2}\left(\frac{n+5}{2}\right) \text {. }
$$

Using definition of $U$ parameter (eq.[48]) we obtain equation (62) as

$$
\Delta U_{G R}=\frac{1}{3}(n+5)
$$

Note that results (A29) and (A33) are not valid for general filters. Equation (A22) that holds for the Gaussian filter plays crucial roles to derive them.

\section{A.3. Generalized Skewness for the Scatter Approach}

First we evaluate the correction term given in equation (A4). With the Fourier space representation (A10) we obtain the following equation

$$
\begin{aligned}
\left\langle\delta_{1}^{2} \nabla^{2} \delta_{2 A}\right\rangle+2\left\langle\delta_{1} \delta_{2 A} \nabla^{2} \delta_{1}\right\rangle= & \int \frac{d \boldsymbol{k}}{(2 \pi)^{3}} \frac{d \boldsymbol{l}}{(2 \pi)^{3}} \frac{d \boldsymbol{m}}{(2 \pi)^{3}} \frac{d \boldsymbol{n}}{(2 \pi)^{3}} \frac{(\boldsymbol{k}+\boldsymbol{l})^{2} R^{2}}{3} \\
& \times \exp \left[-\frac{\left(2 \boldsymbol{l}^{2}+\boldsymbol{k}^{2}+\boldsymbol{m}^{2}+\boldsymbol{n}^{2}+2 \boldsymbol{k} \cdot \boldsymbol{l}\right) R^{2}}{2}\right] \\
& \times\left\langle\delta_{1}(\boldsymbol{k}) \delta_{1}(\boldsymbol{l}) \delta_{1}(\boldsymbol{m}) \delta_{1}(\boldsymbol{n})\right\rangle \exp [i(\boldsymbol{k}+\boldsymbol{l}+\boldsymbol{m}+\boldsymbol{n}) \cdot \boldsymbol{x}], \\
& \times\left[(\boldsymbol{k}+\boldsymbol{l})^{2}+\boldsymbol{m}^{2}+\boldsymbol{n}^{2}\right]
\end{aligned}
$$

With the formula (A13), this twelfth-dimensional integral becomes

$$
\frac{2}{3} \int \frac{d \boldsymbol{k}}{(2 \pi)^{3}} \frac{d \boldsymbol{l}}{(2 \pi)^{3}} \exp \left[-\frac{\left(3 \boldsymbol{l}^{2}+2 \boldsymbol{k}^{2}+2 \boldsymbol{k} \cdot \boldsymbol{l}\right) R^{2}}{2}\right] P(k) P(l)(\boldsymbol{k}+\boldsymbol{l})^{2} R^{2}\left[(\boldsymbol{k}+\boldsymbol{l})^{2}+\boldsymbol{l}^{2}+\boldsymbol{k}^{2}\right] .
$$

changing variables from $d \boldsymbol{k} d \boldsymbol{l}$ to $d k d l d u$ as shown in relation (A21), we obtain the result essentially same as equation (65) as

$$
\begin{aligned}
\left\langle\delta_{1}^{2} \nabla^{2} \delta_{2 A}\right\rangle+2\left\langle\delta_{1} \delta_{2 A} \nabla^{2} \delta_{1}\right\rangle= & \frac{1}{6 \pi^{4}} \int_{0}^{\infty} d k \int_{0}^{\infty} d l \int_{-1}^{1} d u \exp \left[-\frac{\left(3 l^{2}+2 k^{2}+2 k l u\right) R^{2}}{2}\right] \\
& \times k^{2} l^{2} P(k) P(l)\left(k^{2}+l^{2}+2 k l u\right)\left(k^{2}+l^{2}+k l u\right) R^{2}, \quad(\mathrm{~A} 36)
\end{aligned}
$$


As in the case of skewness parameter, we can evaluate this complicated integrals with mathematica and obtain equation (67).

In the same manner, equation (A5) is written as

$$
\begin{aligned}
\left\langle\nabla \delta_{1} \cdot \nabla \delta_{1} \nabla^{2} \delta_{2 A}\right\rangle+2\left\langle\nabla \delta_{1} \nabla \delta_{2 A} \nabla^{2} \delta_{1}\right\rangle= & \int \frac{d \boldsymbol{k}}{(2 \pi)^{3}} \frac{d \boldsymbol{l}}{(2 \pi)^{3}} \frac{d \boldsymbol{m}}{(2 \pi)^{3}} \frac{d \boldsymbol{n}}{(2 \pi)^{3}} \frac{(\boldsymbol{k}+\boldsymbol{l})^{2} R^{2}}{3} \\
& \times \exp \left[-\frac{\left(2 \boldsymbol{l}^{2}+\boldsymbol{k}^{2}+\boldsymbol{m}^{2}+\boldsymbol{n}^{2}+2 \boldsymbol{k} \cdot \boldsymbol{l}\right) R^{2}}{2}\right] \\
& \times\left\langle\delta_{1}(\boldsymbol{k}) \delta_{1}(\boldsymbol{l}) \delta_{1}(\boldsymbol{m}) \delta_{1}(\boldsymbol{n})\right\rangle \\
& \times \exp [i(\boldsymbol{k}+\boldsymbol{l}+\boldsymbol{m}+\boldsymbol{n}) \cdot \boldsymbol{x}], \\
& \times\left[-(\boldsymbol{m} \cdot \boldsymbol{n})(\boldsymbol{k}+\boldsymbol{l})^{2}\right. \\
& \left.-\left(\boldsymbol{m} \cdot(\boldsymbol{k}+\boldsymbol{l}) \boldsymbol{n}^{2}\right)-\left(\boldsymbol{n} \cdot(\boldsymbol{k}+\boldsymbol{l}) \boldsymbol{m}^{2}\right)\right] .(\mathrm{A} 3
\end{aligned}
$$

This expression is simplified to the following form

$$
\begin{aligned}
& \frac{1}{3} \int \frac{d \boldsymbol{k}}{(2 \pi)^{3}} \frac{d \boldsymbol{l}}{(2 \pi)^{3}}(\boldsymbol{k}+\boldsymbol{l})^{2} R^{2} \exp \left[-\frac{\left(2 \boldsymbol{l}^{2}+\boldsymbol{k}^{2}+\boldsymbol{m}^{2}+\boldsymbol{n}^{2}+2 \boldsymbol{k} \cdot \boldsymbol{l}\right) R^{2}}{2}\right] \\
& \times P(k) P(l)\left[4 k^{2} l^{2}-4(\boldsymbol{k} \cdot \boldsymbol{l})^{2}\right] .
\end{aligned}
$$

Again, changing variables, we obtain the expression (66) as

$$
\begin{aligned}
\left\langle\nabla \delta_{1} \cdot \nabla \delta_{1} \nabla^{2} \delta_{2 A}\right\rangle+2\left\langle\nabla \delta_{1} \nabla \delta_{2 A} \nabla^{2} \delta_{1}\right\rangle= & \frac{1}{6 \pi^{4}} \int_{0}^{\infty} d k \int_{0}^{\infty} d l \int_{-1}^{1} d u\left(1-u^{2}\right) R^{2} \\
& \times \exp \left[-\frac{\left(3 l^{2}+2 k^{2}+2 k l u\right) R^{2}}{2}\right] \\
& \times k^{4} l^{4} P(k) P(l)\left(k^{2}+l^{2}+2 k l u\right) .
\end{aligned}
$$

For power-law models we can evaluate this integral using mathematica and obtain equation (68). 


\section{REFERENCES}

Adler, R. J. 1981, The Geometry of Random Fields (Chichester: Wiley)

Babul, A. \& Starkman, G. D. 1992, ApJ, 401, 28

Baugh, C. M, Gaztañaga, E. \& Efstathiou, G. 1995, MNRAS, 274, 1049

Bardeen, J. M., Bond, J. R., Kaiser, N. \& Szalay, A S. 1986, ApJ, 305, 15

Bernardeau, F. 1994, A\&A, 291, 697

Bernardeau, F. \& van de Weygaert, R. 1996, MNRAS, 279, 693

Bouchet, F. Juszkiewicz, R. Colombi, S. \& Pellat, R. 1992, ApJ, 394, L5

Bouchet, F. et al. 1993, ApJ, 417, 36

Canavezes, A. et al. 1998, MNRAS, 297, 17

Coles, P., Davies, A. \& Pearson, R. C. 1996, MNRAS, 281, 1375

Coles, P., Pearson, R. C., Borgani, S. Plionis, M. \& Moscardini, L. 1998, MNRAS, 294, 245

Colless, M. 1998, Phil. Trans. R. Soc. Lond. A. in press (astro-ph/9804079)

Colombi, S., Szapudi, I. \& Szalay, A. S. 1998, MNRAS, 296, 253

Davis, M. \& Peebles, P. J. E. 1983, ApJ, 267, 465

Dekel, A., 1994, ARA\&A, 32, 371

Dekel, A. \& Lahav, O. 1999, ApJ, 520, 24

Doroshkevich, A. G. 1970, Astrofizika, 6, 581 (English transl. Astrophysics, 6, 320)

Fry, J. N. 1984, ApJ, 279, 499

Gaztañaga, E. 1992, ApJ, 398, L17

Goroff, M. H., Grinstein, B., Rey, S. -J., \& Wise, M. B., 1986, ApJ, 311, 6

Gott, J. R., Melott, A. L. \& Dickinson, M. 1986, ApJ, 306, 341

Gott, J. R., Weinberg, D. H. \& Melott, A. L. 1987, ApJ, 319, 1

Gott, J. R. et al. 1989, ApJ, 340, 625 
Gunn, J. E. \& Weinberg, D. H. 1995, in Wide-Field Specroscopy and the Distant Universe eds. S.J. Maddox and A. Aragon-Salamanca.(World Sientific: Singapore), 3

Guth, A. \& Pi, S.-Y. 1982, Phys. Rev. Lett., 49, 1110

Hamilton, A. J. S., Gott, J. R. \& Weinberg, D. H. 1986, ApJ, 309, 1

Hamilton, A. J. S. 1988, PASP, 100, 1343

Hawking, S. W. 1982, Phys. Lett., 115B, 295

Hernquist, L. \& Katz, N. 1989, ApJS, 70, 419

Hivon, E., Bouchet, F. R., Colombi, S., \& Juszkiewicz, R. 1995, A\&A, 298, 643

Juszkiewicz, R., Bouchet, F. R., \& Colombi, S. 1993, ApJ, 412, L9

Juszkiewicz, R. et al. 1995, ApJ, 442, 39

Kaiser, N. 1984, ApJ, 284, L9

Kerscher, M. et al. 1997, MNRAS, 284, 73

Kim, R. S. J., \& Strauss, M. A. 1998, ApJ, 493, 39

Klypin, A. A. 1988, ActaCosmologica, 15, 101

Łokas, E. L., Juszkiewicz, R., Weinberg, D. H. \& Bouchet, F. R. 1995, MNRAS, 274, 730

Luo, S. \& Vishniac, E. T. 1995, ApJS, 96, 429

Matsubara, T. 1994, ApJ, 434, L43

Matsubara, T. 1995, Ph.D. Thesis, Hiroshima Univ

Matsubara, T. \& Suto, Y. 1996, ApJ, 460, 51

Matsubara, T. \& Yokoyama, J. 1996, ApJ, 463, 409

Mecke, K. R., Buchert, T. \& Wagner, H. 1994, A\&A, 288, 697

Melott, A. L., Weinberg, D. H., \& Gott, J. R. 1988, ApJ, 328, 50

Minkowski, H. 1903, Math. Ann., 57, 447

Moore, B. et al. 1992, MNRAS, 256, 477 
Monaghan, J. J. \& Lattanzio, J. J. 1985, A\&A, 149, 135

Okun, B. L. 1990, J. Stat. Phys., 59, 523

Park, C. \& Gott, J. R. 1991, ApJ, 378, 457

Park, C., Gott, J. R., \& da Costa, L. N. 1992, ApJ, 392, L51

Protogeros, Z. A. M. \& Weinberg D. H., 1997, ApJ, 489, 457

Peebles, P. J. E. 1974, A\&A, 32, 197

Peebles, P. J. E. 1980, The Large Scale Structure of the Universe (Princeton University Press: Princeton)

Rhoads, J. E., Gott, J. R., \& Postman, M. 1994, ApJ, 421, 1

Ryden, B. S. 1988, ApJ, 333, L41

Ryden, B. S. et al. 1989, ApJ, 340, 647

Sahni, V. Sathyprakash,B. S. \& Shandarin, S. F. 1997, ApJ, 467, L1

Schmalzing, J. \& Buchert, T. 1997, ApJ, 482, L1

Scoccimarro, R. 1998, MNRAS, 299, 1097

Seto, N., Yokoyama, J., Matsubara, T. \& Siino, M. 1997, ApJS, 110, 177

Seto, N. 1999, ApJ, 523, 24

Springel, V. et al. 1998, MNRAS, 298, 1169

Starobinsky, A. A. 1982, Phys. Lett., 117B, 175

Strauss, M. A. \& Willick, J. A. 1995, Phys. Rep., 261, 271

Thomas, P. A. \& Couchman, H. M. P. 1992, MNRAS, 257, 11

Totsuji, H \& Kihara, T. 1969, PASJ, 21221

Vogeley, M. S. et al. 1994, ApJ, 420, 525

Weinberg, D. H., Gott, J. R., \& Melott, A. L. 1987, ApJ, 321, 2

Weinberg, D. H. \& Cole, S. 1992, MNRAS, 259, 652 
Wolfram, S. 1996, The Mathematica Book, 3rd ed. (Cambridge University Press: Cambridge)

Zeldovich, Ya. B. 1970, A\&A, 5, 84 
TABLE 1

SKEWNESS FOR THE GATHER APPROACH (GAUSSIAN FILTER)

\begin{tabular}{cccccc}
\hline \hline spectral index $n$ & 1 & 0 & -1 & -2 & -3 \\
\hline$S_{F}(n)$ & 3.029 & 3.144 & 3.468 & 4.022 & 4.857 \\
$\Delta S_{G}(n)$ & 4.000 & 3.000 & 2.000 & 1.000 & 0 \\
$S_{G}(n)$ & 7.029 & 6.144 & 5.468 & 5.022 & 4.857 \\
\hline
\end{tabular}

TABLE 2

SKEWNESS FOR THE SCATTER APPROACH

\begin{tabular}{cccccc}
\hline \hline spectral index $n$ & 1 & 0 & -1 & -2 & -3 \\
\hline$S_{F}(n)$ & 3.029 & 3.144 & 3.468 & 4.022 & 4.857 \\
$\Delta_{S} S(n)$ & 3.031 & 2.576 & 2.045 & 1.277 & 0 \\
$S_{S}(n)$ & 6.060 & 5.720 & 5.513 & 5.299 & 4.857 \\
\hline
\end{tabular}

TABLE 3

GENERALIZED SKEWNESS FOR THE GATHER APPROACH

\begin{tabular}{cccccc}
\hline \hline spectral index $n$ & 1 & 0 & -1 & -2 & -3 \\
\hline$T_{F}(n)$ & 2.020 & 2.096 & 2.312 & 2.681 & 3.238 \\
$\Delta T_{G}(n)$ & 3.333 & 2.667 & 2.000 & 1.333 & 0.667 \\
$T_{G}(n)$ & 5.353 & 4.763 & 4.312 & 4.014 & 3.905 \\
\hline$U_{G}(n)$ & 1.431 & 1.292 & 1.227 & 1.222 & 1.272 \\
$\Delta U_{G}(n)$ & 2.000 & 1.667 & 1.333 & 1.000 & 0.667 \\
$U_{G}(n)$ & 3.431 & 2.959 & 2.560 & 2.222 & 1.929 \\
\hline
\end{tabular}

TABLE 4

GENERALIZED SKEWNESS FOR THE SCATTER APPROACH

\begin{tabular}{cccccc}
\hline \hline spectral index $n$ & 1 & 0 & -1 & -2 & -3 \\
\hline$T_{F}(n)$ & 2.020 & 2.096 & 2.312 & 2.681 & 3.238 \\
$\Delta T_{S}(n)$ & -2.082 & -1.908 & -1.723 & -1.451 & -0.963 \\
$T_{S}(n)$ & -0.0623 & 0.1882 & 0.5892 & 1.230 & 2.275 \\
\hline$U_{F}(n)$ & 1.431 & 1.292 & 1.227 & 1.222 & 1.272 \\
$\Delta U_{S}(n)$ & -1.265 & -1.145 & -1.027 & -0.8916 & -0.7105 \\
$U_{S}(n)$ & 0.1662 & 0.1474 & 0.2000 & 0.3301 & 0.5611 \\
\hline
\end{tabular}


Fig. 1
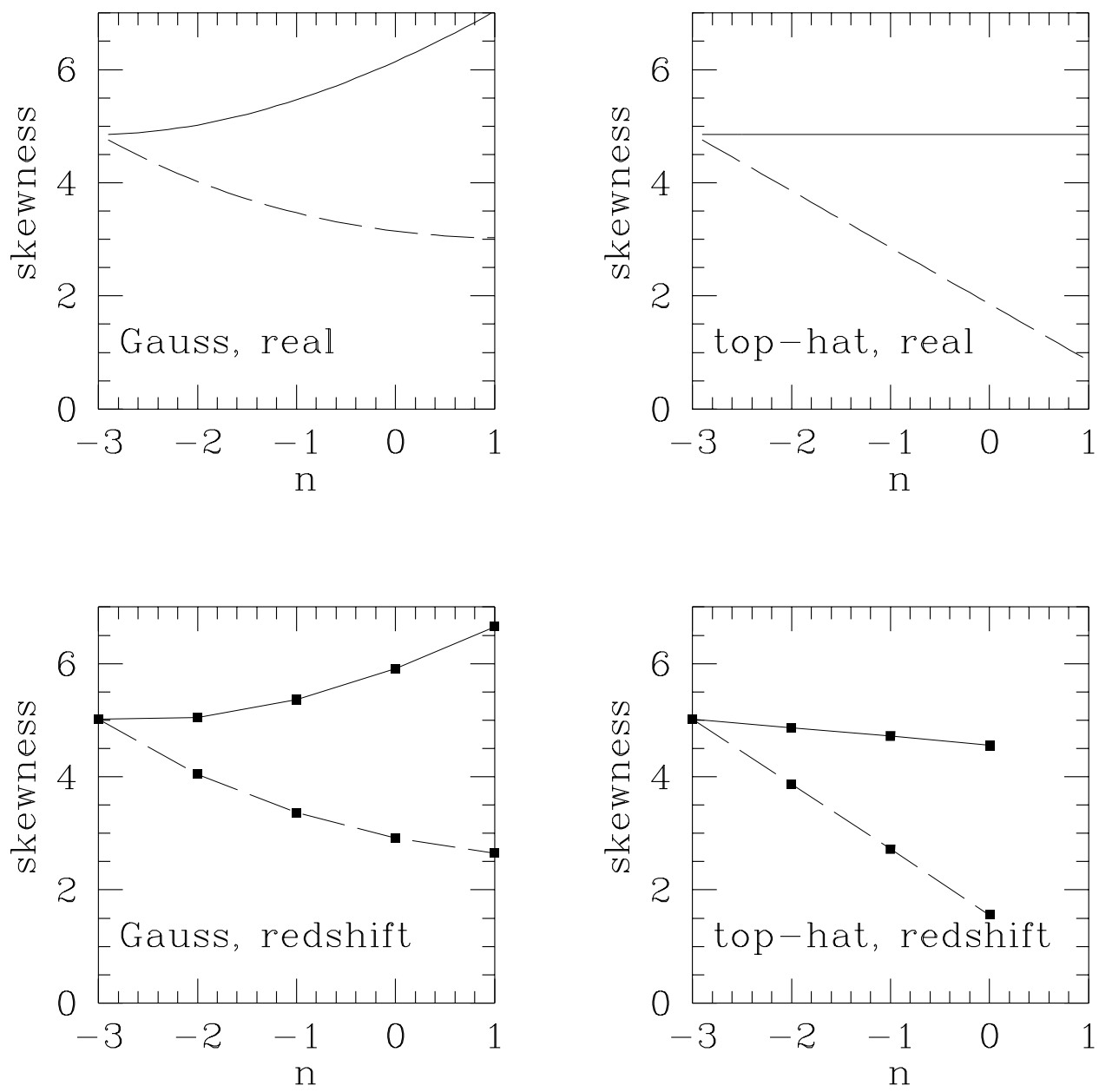

Fig. 1. - Skewness for the gather approach in real and redshift spaces. We use two kinds of filters (Gaussian and top-hat filters). The dashed-lines represent results for the traditional fixed smoothing method and the solid lines for the gather approach. Numerical data in the redshift space are based on Table 2 and 3 of Hivon et al. (1995). 
Fig.2

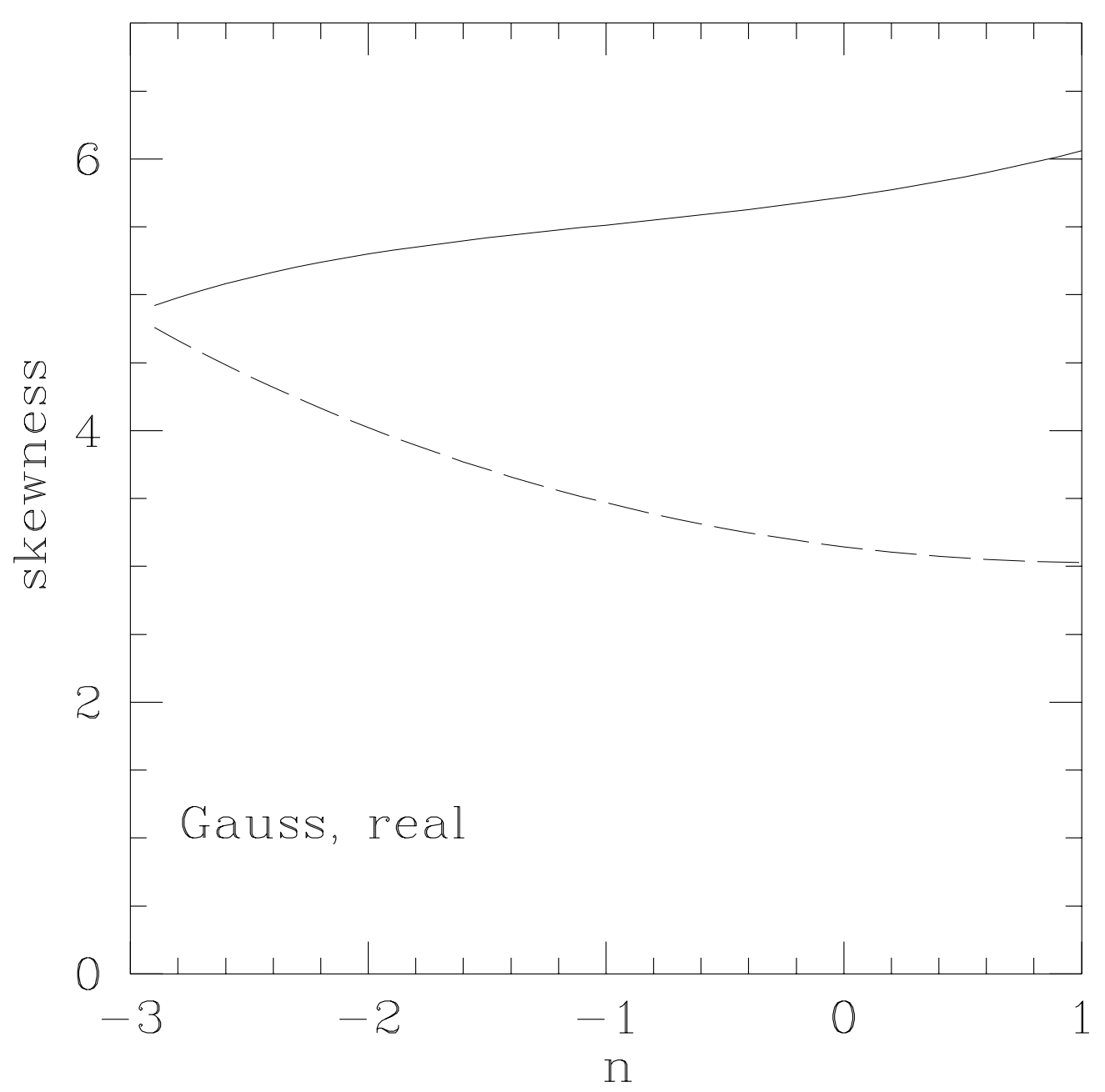

Fig. 2.- Real space skewness for the scatter approach with the Gaussian filters. The dashed-line represents results for the fixed smoothing method and the solid line for the scatter approach. 
Fig.3
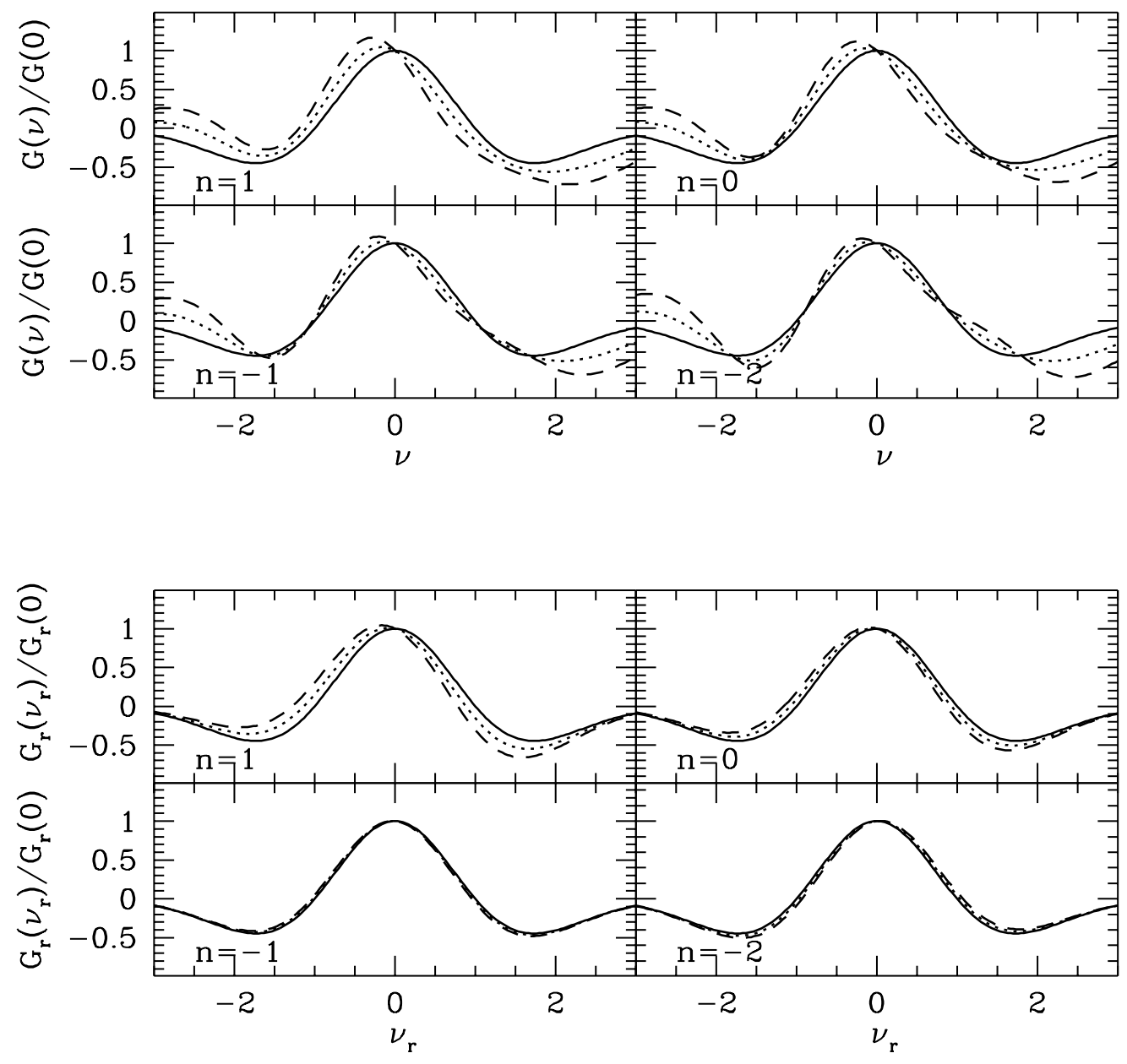

Fig. 3.- Normalized genus density for the fixed Gaussian smoothing (see Matsubara 1994). Upper panel corresponds to $\nu$ - parameterization and lower to $\nu_{r^{-}}$parameterization. The solid curves represent linear curves (eq.[45]). Dotted-lines, dashed-lines show $\sigma=0.2$, and $\sigma=0.4$ respectively. 

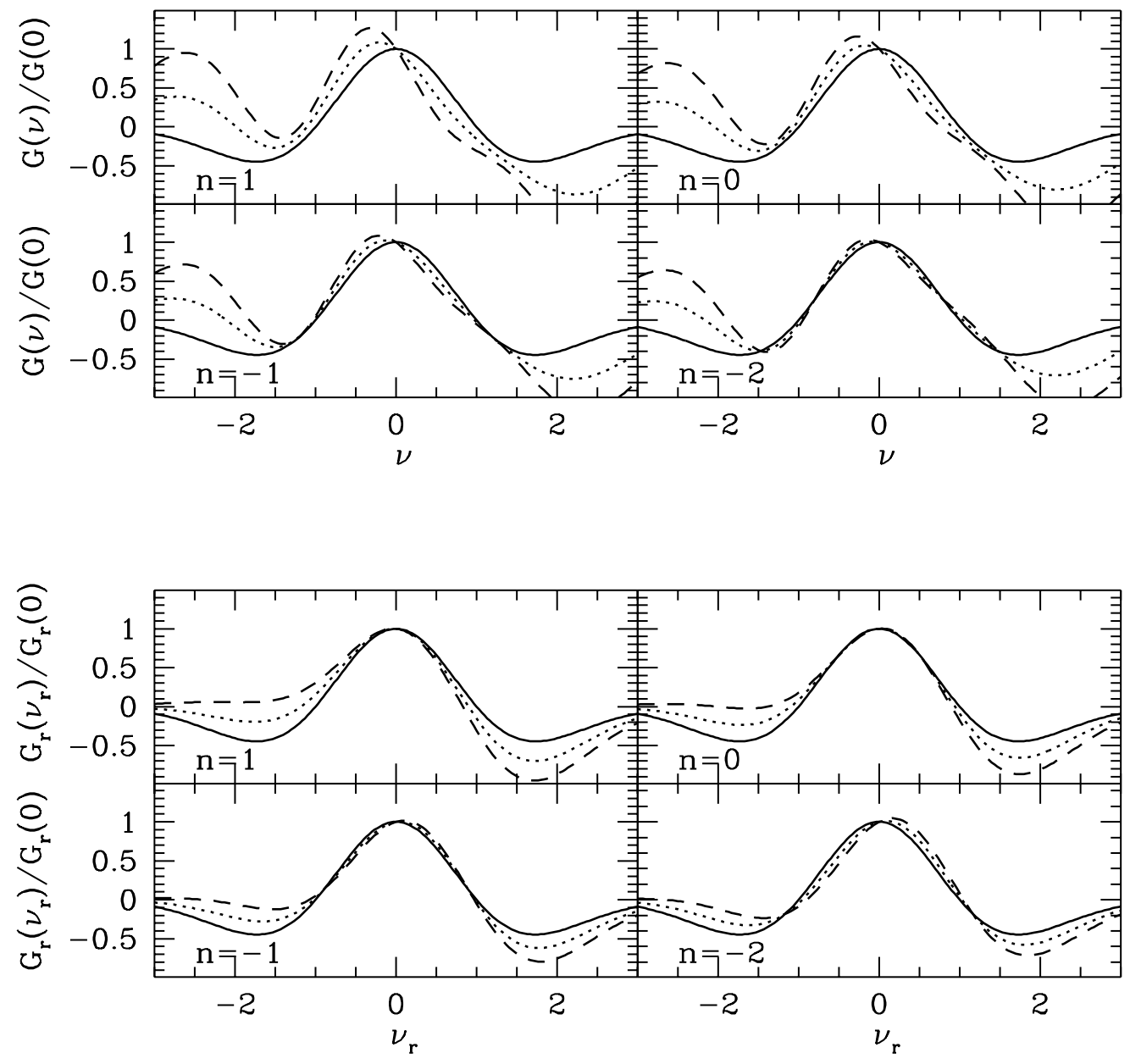

Fig. 4.- Same as Fig.3 but with the gather approach. 
Fig.5
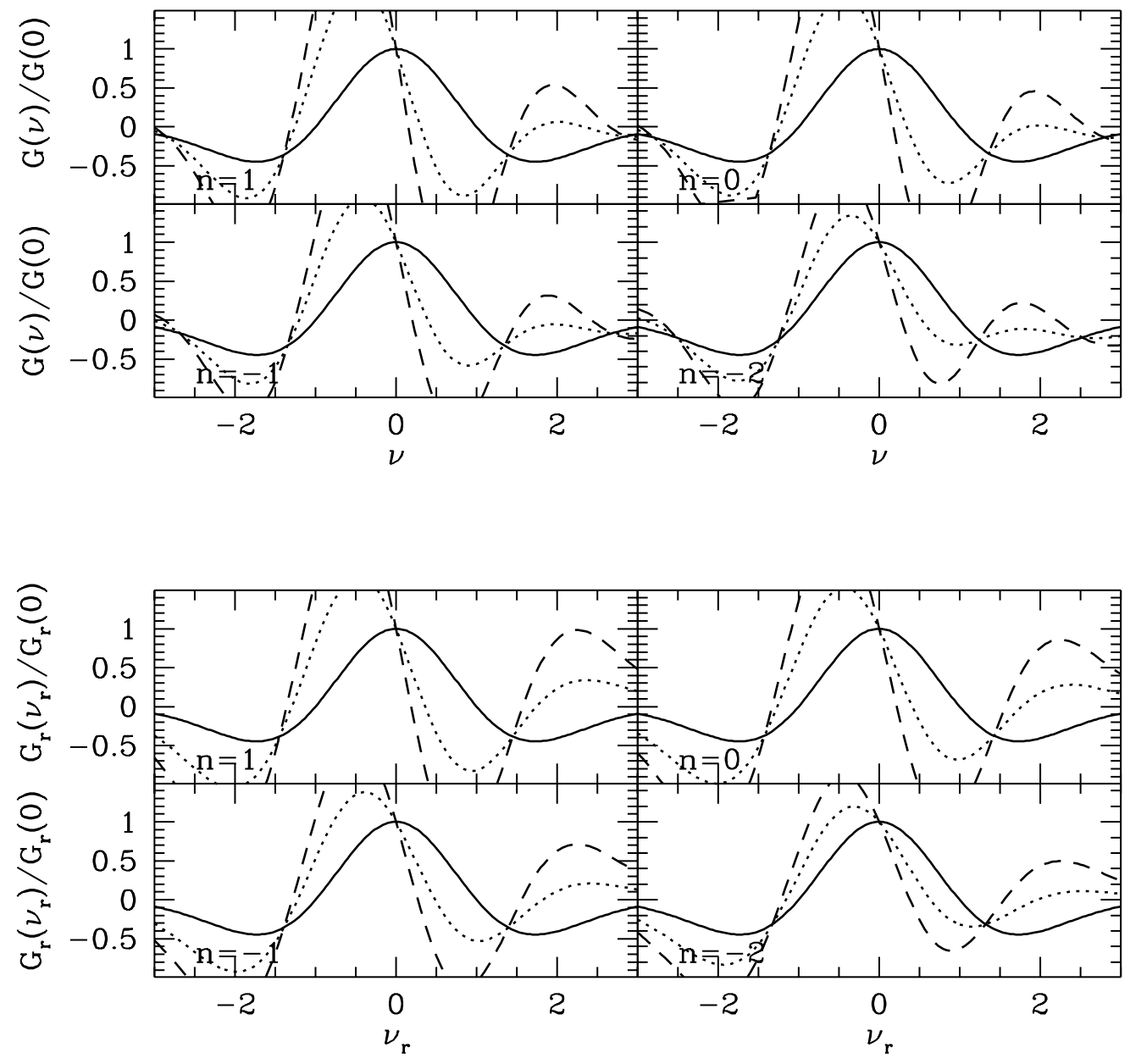

Fig. 5.- Same as Fig.3 but with the scatter approach. 
Fig.6

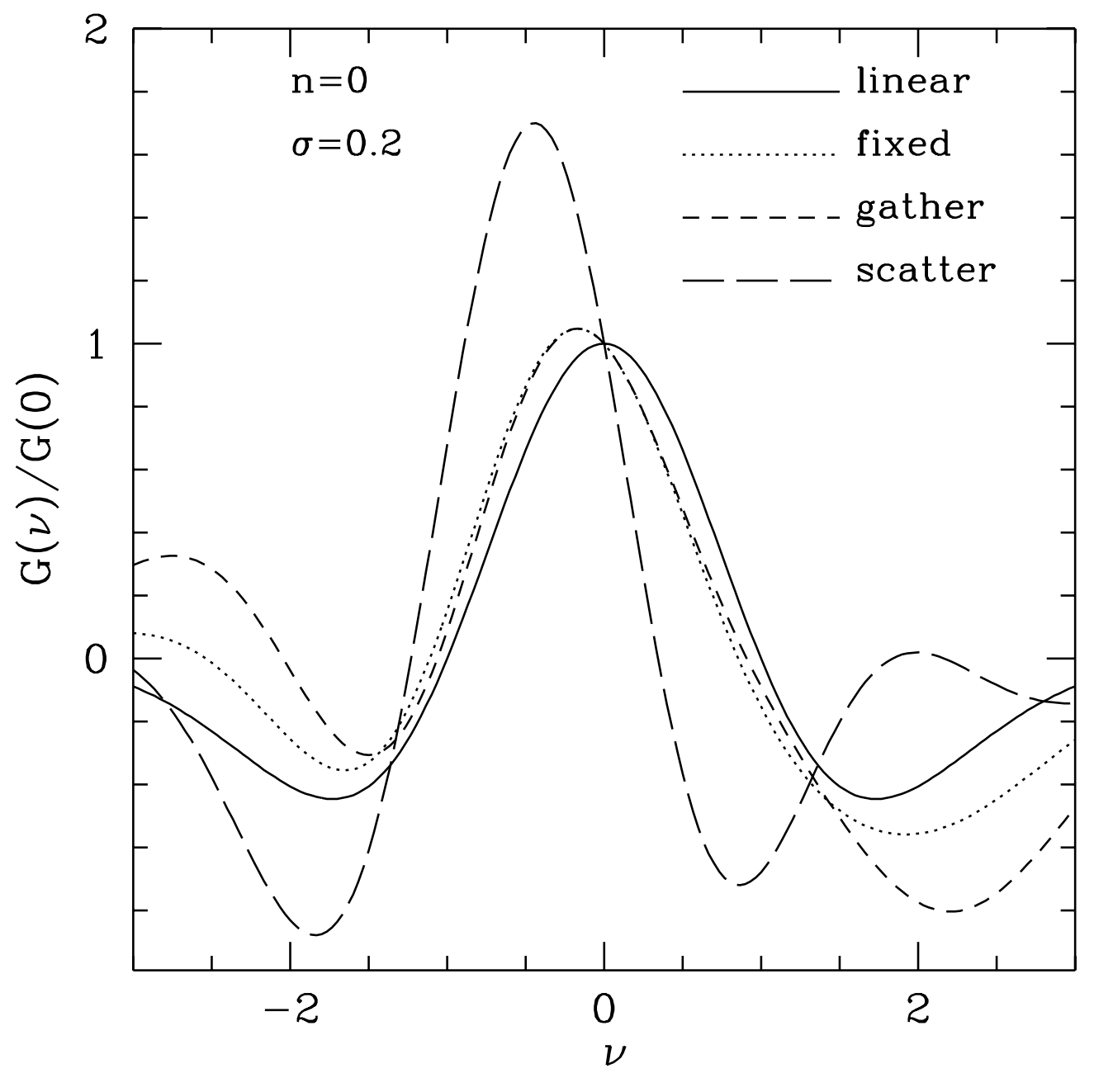

Fig. 6.- Nonlinear Genus curves for various smoothing methods. The solid line corresponds to the linear analysis, dotted to the fixed smoothing, short-dased to the gather approach, and long-dased to the scatter approach. We fix the spectral index at $n=0$ and nonlinearity at $\sigma=0.2$. 
Fig.7
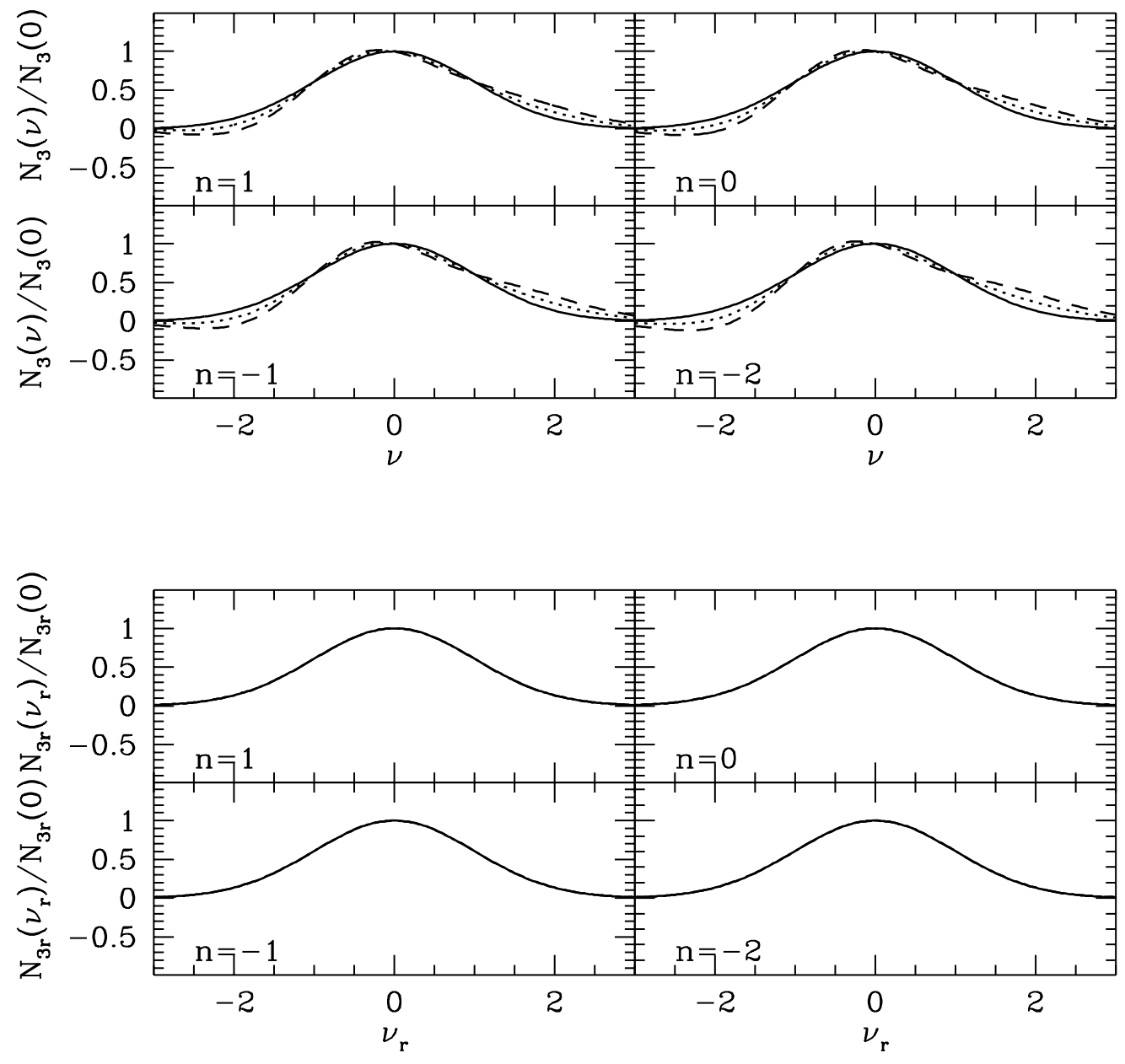

Fig. 7.- Normalized area statistics for the fixed Gaussian smoothing. Upper panel corresponds to $\nu$ parameterization and lower to $\nu_{r}$ parameterization. The solid curves represent the linear prediction (eq.[45]). Dotted-lines, dashed-lines show $\sigma=0.2$, and $\sigma=0.4$ respectively. 
Fig. 8
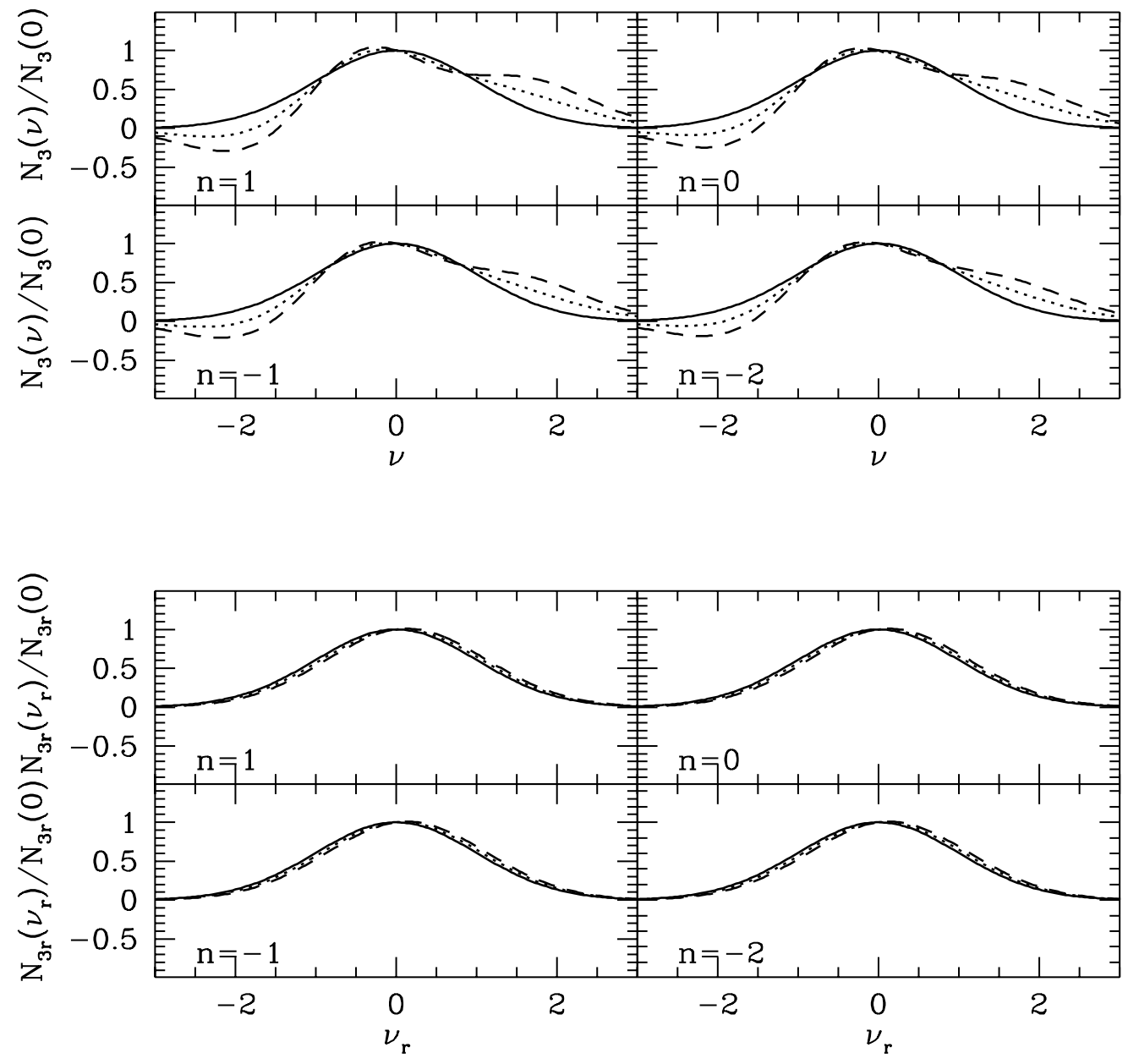

Fig. 8.- Same as Fig.6 but for the gather approach. 
Fig. 9
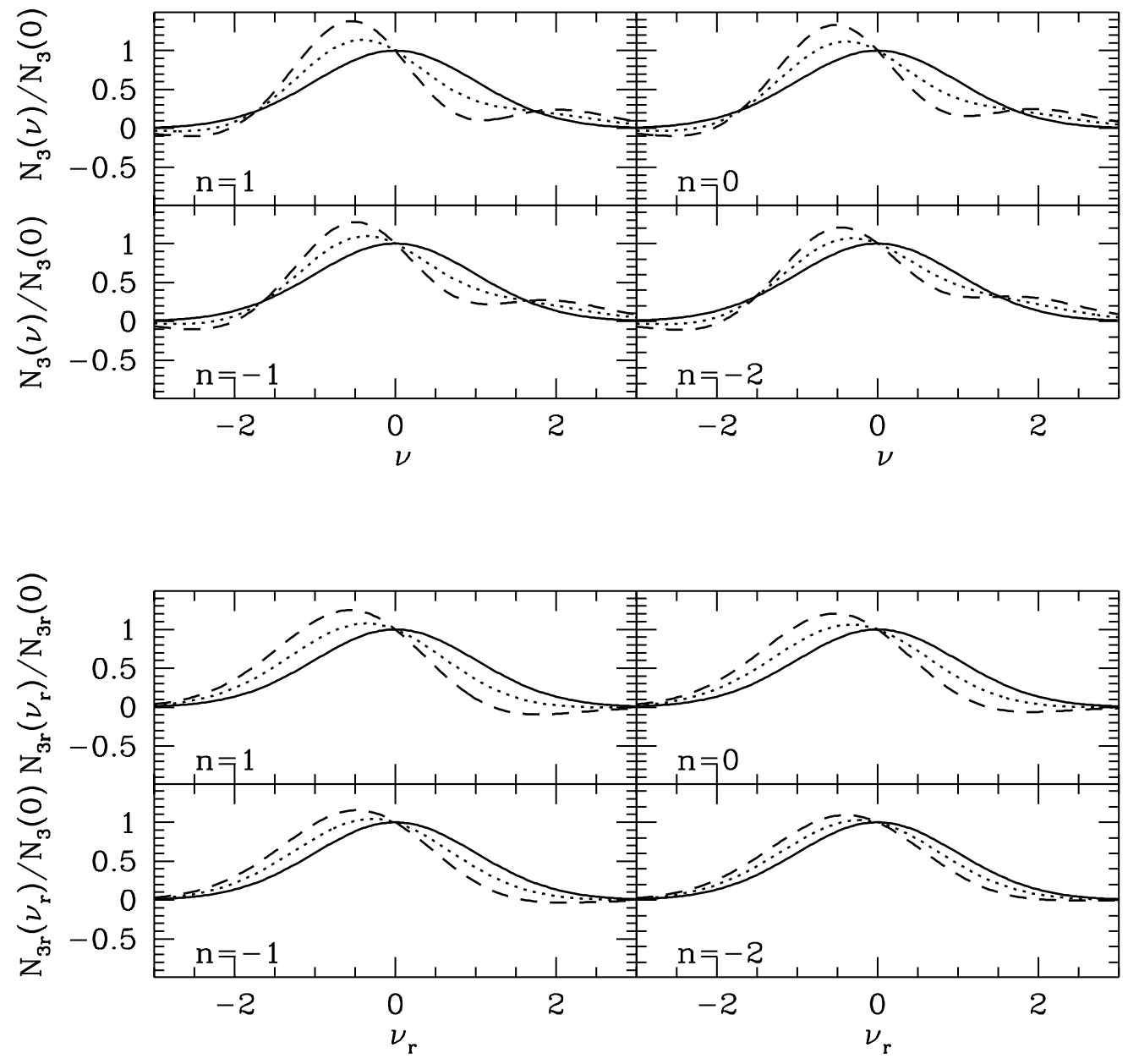

Fig. 9.- Same as Fig.6 but for the scatter approach. 